\title{
Exploring Steric Effects in Diastereoselective Synthesis of Chiral Aminophenolate Zinc Complexes and Stereoselective Ring-Opening Polymerization of rac-Lactide
}

\author{
Haobing Wang, Yang Yang and Haiyan Ma*
}

Shanghai Key Laboratory of Functional Materials Chemistry and Laboratory of Organometallic Chemistry, East China University of Science and Technology, Shanghai (P.R. China).

\section{Contents:}

1. Synthesis of materials

1.1 Synthesis of $(S)$ - $N$-alkyl-prolinamide

1.2 Synthesis of (S)-(1-alkylpyrrolidin-2-yl)methanamine

1.3 Synthesis of (S)-N-alkyl-1-(1-alkylpyrrolidin-2-yl)methanamine

2. X-Ray Diffraction Studies

Tables S1-S2. Crystallographic data and structure refinement for complexes $\mathbf{1}, \mathbf{3 a}, \mathbf{5 a} / \mathbf{b}, \mathbf{7 a}, 10 \mathbf{b}$ and 11

3. ${ }^{1} \mathrm{H}$ NMR and ${ }^{13} \mathrm{C}$ NMR Spectra of Complexes 1-11

Figures S1-S22. ${ }^{1} \mathrm{H}$ NMR and ${ }^{13} \mathrm{C}$ NMR spectra of complexes 1-11

4. MALDI-TOF mass spectra of typical polymer samples

Figures S26-S27. MALDI-TOF mass spectra

5. NMR studies of the reaction of zinc complexes and alcohols

Figures S28-S29. ${ }^{1} \mathrm{H}$ NMR spectra of complexes with alcohols.

6. Microstructure Analysis of Poly(rac-lactide)s

Figures S30-S38. Methine region of homonuclear decoupled ${ }^{1} \mathrm{H}$ NMR spectra of PLAs 


\section{Synthesis of materials ${ }^{\mathrm{S} 1}$}

\subsection{Synthesis of $(S)$ - $N$-alkyl-prolinamide}

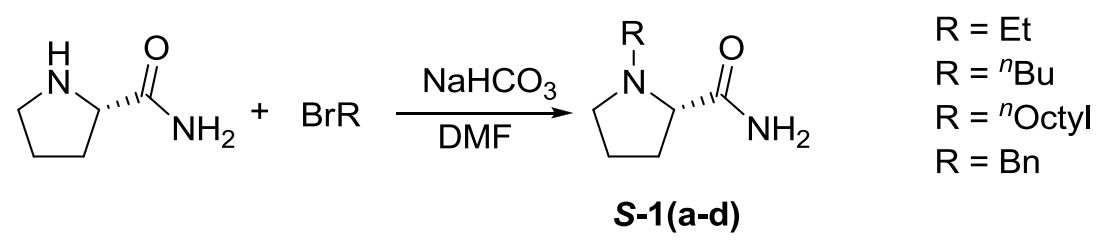

In a typical $\mathrm{N}$-alkylation procedure, a solution of $(S)$-prolinamide $(45.6 \mathrm{~g}, 400 \mathrm{mmol})$ in anhydrous DMF $(200 \mathrm{~mL})$ was placed into a 500-mL round-bottom flask containing a magnetic stirring bar. Bromoethane $(48.0 \mathrm{~g}, 440 \mathrm{mmol})$ and $\mathrm{NaHCO}_{3}(42 \mathrm{~g}, 0.50 \mathrm{mmol})$ were also added to the flask and the reaction mixture was heated at $90{ }^{\circ} \mathrm{C}$ for $3 \mathrm{~h}$. The solvent was subsequently removed under reduced pressure to provide a solid residue that was washed with warm $\mathrm{CH}_{2} \mathrm{Cl}_{2}(250$ $\mathrm{mL} \times 2)$. The organic solution was dried $\left(\mathrm{Na}_{2} \mathrm{SO}_{4}\right)$ and the solvent was removed under reduced pressure to provide the alkylated product. This material was passed through a column of silica (EA) and provided $N$-ethylprolinamide $\left(22.7 \mathrm{~g}, 40 \%\right.$ ), S-1a: ${ }^{1} \mathrm{H}$ NMR (400 $\mathrm{MHz}, \mathrm{CDCl}_{3}$ ): $\delta 7.20$ (br s, $1 \mathrm{H}, \mathrm{CONH}_{2}$ ), 5.92 (br s, $\left.1 \mathrm{H}, \mathrm{CONH}_{2}\right), 3.21-3.10\left(\mathrm{~m}, 1 \mathrm{H}, \mathrm{N}-\mathrm{CH}_{2}\right.$ of pyrrolidinyl), 2.95 (dd, ${ }^{2} \mathrm{~J}=$ $10.3,{ }^{3} \mathrm{~J}=4.9 \mathrm{~Hz}, 1 \mathrm{H}, \mathrm{N}-\mathrm{CH}$ of pyrrolidinyl), $2.66\left(\mathrm{dq},{ }^{2} \mathrm{~J}=12.0,{ }^{3} \mathrm{~J}=7.2 \mathrm{~Hz}, 1 \mathrm{H}, \mathrm{NCH}_{2} \mathrm{CH}_{3}\right), 2.44$ (dq, ${ }^{2} J=12.0,{ }^{3} J=7.2 \mathrm{~Hz}, 1 \mathrm{H}, \mathrm{NCH}_{2} \mathrm{CH}_{3}$ ), 2.26 (m, 1H, N-CH $\mathrm{H}_{2}$ of pyrrolidinyl), 2.19-2.07 (m, 1H, $-\mathrm{CH}_{2-}$ of pyrrolidinyl), $1.90-1.80\left(\mathrm{~m}, 1 \mathrm{H},-\mathrm{CH}_{2^{-}}\right.$of pyrrolidinyl), $1.78-1.66\left(\mathrm{~m}, 2 \mathrm{H},-\mathrm{CH}_{2^{-}}\right.$of pyrrolidinyl), $1.05\left(\mathrm{t},{ }^{3} \mathrm{~J}=7.2 \mathrm{~Hz}, 3 \mathrm{H}, \mathrm{NCH}_{2} \mathrm{CH}_{3}\right)$.

S-1b, $N$ - ${ }^{n}$ butylprolinamide $(34.0 \mathrm{~g}, 50 \%):{ }^{1} \mathrm{H}$ NMR $\left(400 \mathrm{MHz}, \mathrm{CDCl}_{3}\right): \delta 7.25$ (br s, $1 \mathrm{H}$, $\left.\mathrm{CON}_{2}\right), 5.80\left(\right.$ br s, $\left.1 \mathrm{H}, \mathrm{CONH}_{2}\right), 3.18-3.14\left(\mathrm{~m}, 1 \mathrm{H}, \mathrm{NCH}_{2^{-}}\right.$of pyrrolidinyl), $2.96\left(\mathrm{dd}, 1 \mathrm{H},{ }^{2} J=\right.$ $10.2 \mathrm{~Hz},{ }^{3} \mathrm{~J}=4.9 \mathrm{~Hz}, \mathrm{NCH}$ - of pyrrolidinyl), 2.66-2.55 (m, 1H, $\mathrm{NCH}_{2^{-}}$of pyrrolidinyl), 2.44-2.35 (m, $\left.1 \mathrm{H}, \mathrm{NCH}_{2} \mathrm{CH}_{2} \mathrm{CH}_{2} \mathrm{CH}_{3}\right), 2.31-2.21\left(\mathrm{~m}, 1 \mathrm{H}, \mathrm{NCH}_{2} \mathrm{CH}_{2} \mathrm{CH}_{2} \mathrm{CH}_{3}\right), 2.21-2.06\left(\mathrm{~m}, 1 \mathrm{H},-\mathrm{CH}_{2-}\right.$ of pyrrolidinyl), 1.91-1.82 (m, $1 \mathrm{H},-\mathrm{CH}_{2}$ of pyrrolidinyl), $1.80-1.67$ (m, $2 \mathrm{H},-\mathrm{CH}_{2^{-}}$of pyrrolidinyl), 1.49-1.17 (m, 4H, $\mathrm{NCH}_{2} \mathrm{CH}_{2} \mathrm{CH}_{2} \mathrm{CH}_{3}$ ), 0.93-0.84 (t, $J=7.2 \mathrm{~Hz}, 3 \mathrm{H}, \mathrm{NCH}_{2} \mathrm{CH}_{2} \mathrm{CH}_{2} \mathrm{CH}_{3}$ ).

S-1c, $N$ - $^{n}$ octylprolinamide (58.6 g, 85\%): ${ }^{1} \mathrm{H}$ NMR (400 MHz, $\left.\mathrm{CDCl}_{3}\right): \delta 7.20$ (br s, $1 \mathrm{H}$, $\left.\mathrm{CON}_{2}\right), 5.75\left(\mathrm{br} \mathrm{s}, 1 \mathrm{H}, \mathrm{CON} H_{2}\right), 3.20-13\left(\mathrm{~m}, 1 \mathrm{H}, \mathrm{NCH}_{2}\right.$ - of pyrrolidinyl), $2.98\left(\mathrm{dd},{ }^{2} J=10.2,{ }^{3} J=\right.$ 
$4.9 \mathrm{~Hz}, 1 \mathrm{H}, \mathrm{NCH}$ - of pyrrolidinyl), $2.62\left(\mathrm{dt},{ }^{2} \mathrm{~J}=11.8,{ }^{3} \mathrm{~J}=8.3 \mathrm{~Hz}, 1 \mathrm{H}, \mathrm{NCH}_{2}\right.$ - of pyrrolidinyl), 2.46-2.36 (m, 1H, $\left.\mathrm{NCH}_{2}\left(\mathrm{CH}_{2}\right)_{6} \mathrm{CH}_{3}\right), 2.23-2.10\left(\mathrm{~m}, 1 \mathrm{H}, \mathrm{NCH}_{2}\left(\mathrm{CH}_{2}\right)_{6} \mathrm{CH}_{3}\right), 1.95-1.83\left(\mathrm{~m}, 2 \mathrm{H},-\mathrm{CH}_{2}-\right.$ of pyrrolidinyl), 1.83-1.69 (m, $2 \mathrm{H},-\mathrm{CH}_{2^{-}}$of pyrrolidinyl), 1.54-1.39 (m, $\left.2 \mathrm{H}, \mathrm{NCH}_{2}\left(\mathrm{CH}_{2}\right)_{6} \mathrm{CH}_{3}\right)$, 1.39-1.17 (m, $\left.10 \mathrm{H}, \mathrm{NCH}_{2}\left(\mathrm{CH}_{2}\right)_{6} \mathrm{CH}_{3}\right), 0.88\left(\mathrm{t},{ }^{3} \mathrm{~J}=6.8 \mathrm{~Hz}, 3 \mathrm{H}, \mathrm{NCH}_{2}\left(\mathrm{CH}_{2}\right)_{6} \mathrm{CH}_{3}\right)$.

S-1d, $N$-benzylprolinamide (70 g, 86\%): ${ }^{1} \mathrm{H}$ NMR (400 MHz, $\left.\mathrm{CDCl}_{3}\right): \delta 7.42-7.14(\mathrm{~m}, 5 \mathrm{H}, \mathrm{Ph}-$ H), 6.27 (br s, $1 \mathrm{H}, \mathrm{CONH}_{2}$ ), $5.60\left(\right.$ br s, $\left.1 \mathrm{H}, \mathrm{CONH}_{2}\right), 3.92\left(\mathrm{~d},{ }^{2} \mathrm{~J}=13.0 \mathrm{~Hz}, 1 \mathrm{H}, \mathrm{NCH}_{2} \mathrm{Ph}\right), 3.43$ (d, $\left.{ }^{2} J=13.0 \mathrm{~Hz}, 1 \mathrm{H}, \mathrm{NCH}_{2} \mathrm{Ph}\right), 3.15\left(\mathrm{dt},{ }^{2} J=11.4,{ }^{3} J=5.7 \mathrm{~Hz}, 1 \mathrm{H}, \mathrm{NCH}_{2}\right.$ - of pyrrolidinyl), 3.06-2.94 (m, $1 \mathrm{H}, \mathrm{NCH}$ - of pyrrolidinyl), 2.36-2.26 (m, 1H, $\mathrm{NCH}_{2}$ - of pyrrolidinyl), $2.20\left(\mathrm{~m}, 1 \mathrm{H},-\mathrm{CH}_{2}-\right.$ of pyrrolidinyl), 1.90 (m, $1 \mathrm{H},-\mathrm{CH}_{2}$ - of pyrrolidinyl), 1.80-1.66 (m, $2 \mathrm{H},-\mathrm{CH}_{2}$ - of pyrrolidinyl).

\subsection{Synthesis of $(S)$-(1-alkylpyrrolidin-2-yl)methanamine}

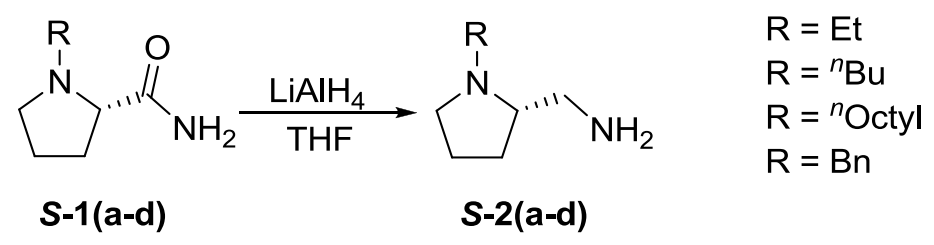

$\mathrm{LiAlH}_{4}(7.6 \mathrm{~g}, 200 \mathrm{mmol})$ and dry THF $(50 \mathrm{~mL})$ were added to a $250 \mathrm{~mL}$ 3-neck flask equipped with condenser, magnetic stir-bar, addition funnel and inlet for argon. A solution of $N$ ethylprolinamide $(14.2 \mathrm{~g}, 100 \mathrm{mmol})$ in $50 \mathrm{~mL}$ of THF was added dropwise over 30 min to the above mixture. The slightly cloudy mixture was stirred at room temperature for $60 \mathrm{~min}$, and then heated under refluxing for $18 \mathrm{~h}$. The resulting opaque milky-white solution was cooled to room temperature and quenched by the careful addition of $10 \mathrm{~mL}$ saturated aqueous $\mathrm{Na}_{2} \mathrm{SO}_{4}$ solution. Inorganic salts were removed by filtration and rinsed liberally with $\mathrm{Et}_{2} \mathrm{O}$. The solvent was removed under reduced pressure to obtain final amine without further purification. S-2a, $(S)-(1$ ethylpyrrolidin-2-yl)methanamine: ${ }^{1} \mathrm{H}$ NMR $\left(400 \mathrm{MHz}, \mathrm{CDCl}_{3}\right): \delta 3.11-2.98\left(\mathrm{~m}, 1 \mathrm{H}, \mathrm{N}-\mathrm{CH} H_{2}\right.$ of pyrrolidinyl), 2.90-2.81 (m, 1H, N-CH of pyrrolidinyl), 2.51-2.34 (m, 3H, $\mathrm{NCH}_{2} \mathrm{CH} \& \mathrm{NCH}_{2} \mathrm{CH}_{3}$ ), $2.22\left(\mathrm{dq},{ }^{2} \mathrm{~J}=12.0,{ }^{3} \mathrm{~J}=7.2 \mathrm{~Hz}, 1 \mathrm{H}, \mathrm{NCH}_{2} \mathrm{CH}_{3}\right), 2.19-2.11\left(\mathrm{~m}, 1 \mathrm{H}, \mathrm{NCH}_{2}\right.$ - of pyrrolidinyl), 2.06- 
$1.95\left(\mathrm{~m}, 1 \mathrm{H},-\mathrm{CH}_{2}\right.$ - of pyrrolidinyl), 1.88-1.76 (m, 1H, - $\mathrm{CH}_{2}$ - of pyrrolidinyl), 1.61-1.50 (m, 2H, $\mathrm{CH}_{2}$ - of pyrrolidinyl), 1.40-1.26 (br s, $\left.1 \mathrm{H}, \mathrm{CH}_{2} \mathrm{NH}_{2}\right), 1.05\left(\mathrm{t},{ }^{3} \mathrm{~J}=7.2 \mathrm{~Hz}, 3 \mathrm{H}, \mathrm{NCH}_{2} \mathrm{CH}_{3}\right.$ ).

S-2b, $(S)-\left(1-{ }^{n}\right.$ butylpyrrolidin-2-yl)methanamine (10.8 g, 69\%). ${ }^{1} \mathrm{H}$ NMR (400 MHz, $\left.\mathrm{CDCl}_{3}\right): \delta$ 3.13-3.09 (m, 1H, $\mathrm{NCH}_{2}$ - of pyrrolidinyl), 2.72-2.62 (m, 3H, $\mathrm{NCH}_{2} \mathrm{CH} \& \mathrm{NCH}$ - of pyrrolidinyl), 2.34-2.29 (m, 1H, $\mathrm{NCH}_{2}$ - of pyrrolidinyl ), 2.15-2.07 (m, $\left.2 \mathrm{H}, \mathrm{NCH}_{2} \mathrm{CH}_{2} \mathrm{CH}_{2} \mathrm{CH}_{3}\right), 1.86-1.81(\mathrm{~m}, 1 \mathrm{H}$, - $\mathrm{CH}_{2^{-}}$of pyrrolidinyl), $1.70-1.67\left(\mathrm{~m}, 2 \mathrm{H},-\mathrm{CH}_{2^{-}}\right.$of pyrrolidinyl), $1.65-1.24\left(\mathrm{~m}, 7 \mathrm{H},-\mathrm{CH}_{2^{-}}\right.$of pyrrolidinyl \& $\left.\mathrm{NCH}_{2} \mathrm{CH}_{2} \mathrm{CH}_{2} \mathrm{CH}_{3} \& \mathrm{CH}_{2} \mathrm{NH}_{2}\right), 0.88\left(\mathrm{t},{ }^{3} \mathrm{~J}=6.8 \mathrm{~Hz}, 3 \mathrm{H}, \mathrm{NCH}_{2} \mathrm{CH}_{2} \mathrm{CH}_{2} \mathrm{CH}_{3}\right)$.

S-2c, $(S)-\left(1-^{n}\right.$ octylpyrrolidin-2-yl)methanamine (15.9 g, 75\%). ${ }^{1} \mathrm{H}$ NMR $\left(400 \mathrm{MHz}, \mathrm{CDCl}_{3}\right): \delta$ $3.12\left(\mathrm{dt}^{2}{ }^{2} \mathrm{~J}=9.3,{ }^{3} \mathrm{~J}=4.6 \mathrm{~Hz}, 1 \mathrm{H}, \mathrm{NCH}_{2}\right.$ - of pyrrolidinyl), $2.76-2.60$ (m, 3H, $\mathrm{NCH}_{2} \mathrm{CH} \& \mathrm{NCH}-$ of pyrrolidinyl), $2.33\left(\mathrm{dt},{ }^{2} \mathrm{~J}=12.2,{ }^{3} \mathrm{~J}=6.4 \mathrm{~Hz}, 1 \mathrm{H}, \mathrm{NCH}_{2^{-}}\right.$of pyrrolidinyl), 2.21-2.01 (m, $2 \mathrm{H}$, $\left.\mathrm{NCH}_{2}\left(\mathrm{CH}_{2}\right)_{6} \mathrm{CH}_{3}\right), 1.91-1.78\left(\mathrm{~m}, 1 \mathrm{H},-\mathrm{CH}_{2-}\right.$ of pyrrolidinyl), 1.78-1.52 (m, 5H, $-\mathrm{CH}_{2^{-}}$of pyrrolidinyl \& $\left.\mathrm{NCH}_{2}\left(\mathrm{CH}_{2}\right)_{6} \mathrm{CH}_{3}\right), 1.51-1.37\left(\mathrm{~m}, 2 \mathrm{H},-\mathrm{CH}_{2}-\right.$ of pyrrolidinyl), $1.30-1.01(\mathrm{~m}, 10 \mathrm{H}$, $\left.\mathrm{NCH}_{2}\left(\mathrm{CH}_{2}\right)_{6} \mathrm{CH}_{3} \& \mathrm{CH}_{2} \mathrm{NH}_{2}\right), 0.86\left(\mathrm{t}, J=6.8 \mathrm{~Hz}, 3 \mathrm{H}, \mathrm{NCH}_{2}\left(\mathrm{CH}_{2}\right)_{6} \mathrm{CH}_{3}\right)$.

S-2d, (S)-(1-benzylpyrrolidin-2-yl)methanamine (14.3 g, 75\%). ${ }^{1} \mathrm{H}$ NMR (400 MHz, $\left.\mathrm{CDCl}_{3}\right)$ : $\delta$ 7.42-7.10 (m, 5H, Ph- $H), 3.92\left(\mathrm{~d},{ }^{2} J=13.2 \mathrm{~Hz}, 1 \mathrm{H}, \mathrm{NCH}_{2} \mathrm{Ph}\right), 3.23\left(\mathrm{~d},{ }^{2} J=13.2 \mathrm{~Hz}, 1 \mathrm{H}\right.$, $\left.\mathrm{NCH}_{2} \mathrm{Ph}\right), 2.95-2.85\left(\mathrm{~m}, 1 \mathrm{H}, \mathrm{NCH}_{2^{-}}\right.$of pyrrolidinyl), 2.75-2.63 (m, 2H, $\mathrm{NCH}_{2} \mathrm{CH} \& \mathrm{NCH}-$ of pyrrolidinyl), 2.55-2.45 (m, $1 \mathrm{H}, \mathrm{NCH}_{2^{-}}$of pyrrolidinyl), $2.14\left(\mathrm{dd},{ }^{2} \mathrm{~J}=11.2,{ }^{3} \mathrm{~J}=4.7 \mathrm{~Hz}, 1 \mathrm{H}\right.$, $\left.\mathrm{NCH}_{2} \mathrm{CH}\right), 1.93-1.80\left(\mathrm{~m}, 1 \mathrm{H},-\mathrm{CH}_{2}\right.$ - of pyrrolidinyl), 1.72-1.56 (m, 3H, $-\mathrm{CH}_{2}$ - of pyrrolidinyl), 1.27 (br s, $2 \mathrm{H}, \mathrm{CH}_{2} \mathrm{NH}_{2}$ ).

\subsection{Synthesis of $(S)$ - $N$-alkyl-1-(1-alkylpyrrolidin-2-yl)methanamine}

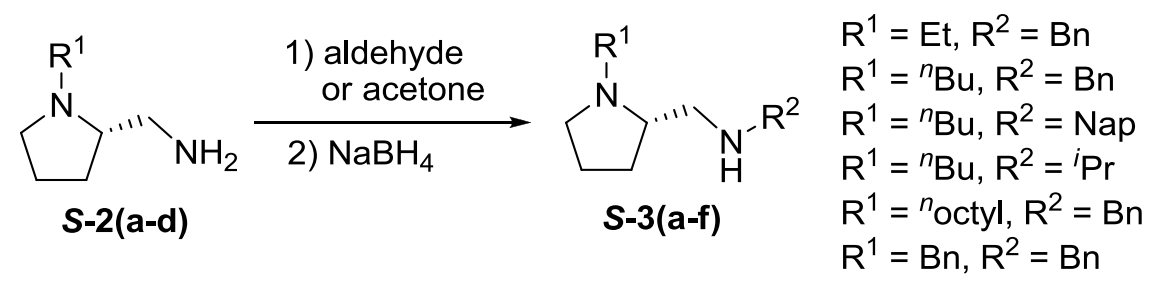

(S)-(1-Ethylpyrrolidin-2-yl)methanamine (1.92 g, $0.015 \mathrm{~mol})$ was added to a solution of benzaldehyde $(1.75 \mathrm{~g}, 0.017 \mathrm{~mol})$ in ethanol $(20 \mathrm{~mL})$ and the mixture was heated to reflux for $24 \mathrm{~h}$. 
After cooling to r.t., sodium borohydride $(1.33 \mathrm{~g}, 0.035 \mathrm{mmol})$ was sequentially added to the above light yellow solution in three times and the mixture was stirred for $3 \mathrm{~h}$ at $60{ }^{\circ} \mathrm{C}$. The mixture was poured into water and extracted with methylene dichloride. The organic phase was dried over anhydrous $\mathrm{MgSO}_{4}$. Evaporation of the solvent gave the target product, $(\boldsymbol{S})-\boldsymbol{N}$-benzyl-1-(1ethylpyrrolidin-2-yl)methanamine, as yellow viscous oil (2.96 g), which was used directly for the next step without further purification.

(S)-N-benzyl-1-(1- ${ }^{n}$ butylpyrrolidin-2-yl)methanamine was synthesized similarly from $(S)$ (1- ${ }^{n}$ butylpyrrolidin-2-yl)methanamine $(1.56 \mathrm{~g}, 1.0 \mathrm{~mol})$, benzaldehyde $(1.17 \mathrm{~g}, 1.1 \mathrm{~mol})$ and sodium borohydride (0.86 g, $2.2 \mathrm{~mol})$. After workup, the resultant yellow viscous oil was used directly for the next step without further purification.

(S)-N-(methyl-1-napthyl)-1-(1- ${ }^{n}$ butylpyrrolidin-2-yl)methanamine was synthesized similarly from $(S)$-(1- ${ }^{n}$ butylpyrrolidin-2-yl)methanamine (1.56 g, $\left.1.0 \mathrm{~mol}\right)$, 1-naphthaldehyde $(1.72$ g, $1.1 \mathrm{~mol})$ and sodium borohydride $(0.86 \mathrm{~g}, 2.2 \mathrm{~mol})$. After workup, the resultant yellow viscous oil was used directly for the next step without further purification.

$(S)-N$ - $^{i}$ propyl-1-(1- ${ }^{n}$ butylpyrrolidin-2-yl)methanamine was synthesized similarly from $(S)$ (1- ${ }^{n}$ butylpyrrolidin-2-yl)methanamine $(1.56 \mathrm{~g}, 1.0 \mathrm{~mol})$, acetone $(1.16 \mathrm{~g}, 2.0 \mathrm{~mol})$ and sodium borohydride (1.72 g, $4.4 \mathrm{~mol}$ ). After workup, the resultant yellow viscous oil was used directly for the next step without further purification.

(S)-N-benzyl-1-(1- ${ }^{n}$ octylpyrrolidin-2-yl)methanamine was synthesized similarly from $(S)$ (1- ${ }^{n}$ octylpyrrolidin-2-yl)methanamine $(2.12 \mathrm{~g}, 1.0 \mathrm{~mol})$, benzaldehyde $(1.17 \mathrm{~g}, 1.1 \mathrm{~mol})$ and sodium borohydride (0.86 g, $2.2 \mathrm{~mol})$. After workup, the resultant yellow viscous oil was used directly for the next step without further purification.

(S)-N-benzyl-1-(1-benzylpyrrolidin-2-yl)methanamine was synthesized similarly from $(S)$ (1-benzylpyrrolidin-2-yl)methanamine (1.90 g, $1.0 \mathrm{~mol})$, benzaldehyde $(1.17 \mathrm{~g}, 1.1 \mathrm{~mol})$ and sodium borohydride $(0.86 \mathrm{~g}, 2.2 \mathrm{~mol})$. After workup, the resultant yellow viscous oil was used directly for the next step without further purification. 


\section{X-Ray Diffraction Studies}

Table S1. Crystallographic data and structure refinement for complexes $\mathbf{1}, \mathbf{3 a}$ and $\mathbf{5 a} / \mathbf{b}$

\begin{tabular}{|c|c|c|c|}
\hline & $1 \mathrm{a}$ & $\mathbf{3 a}$ & $5 \mathbf{a} / \mathbf{b}$ \\
\hline Empirical formula & $\mathrm{C}_{29} \mathrm{H}_{47} \mathrm{Cl}_{2} \mathrm{~N}_{3} \mathrm{OSi}_{2} \mathrm{Zn}$ & $\mathrm{C}_{37} \mathrm{H}_{65} \mathrm{~N}_{3} \mathrm{OSi}_{2} \mathrm{Zn}$ & $\mathrm{C}_{52} \mathrm{H}_{63} \mathrm{~N}_{3} \mathrm{OSi}_{2} \mathrm{Zn} \cdot \mathrm{C}_{7} \mathrm{H}_{8}$ \\
\hline Formula weight & 646.19 & 689.47 & 959.73 \\
\hline Temp (K) & $140(2)$ & $293(2)$ & $140(2)$ \\
\hline Crystal size (mm) & $0.300 \times 0.180 \times 0.090$ & $0.254 \times 0.211 \times 0.147$ & $0.300 \times 0.150 \times 0.050$ \\
\hline Crystal system & Orthorhombic & Monoclinic & Triclinic \\
\hline Space group & $P 2(1) 2(1) 2(1)$ & $P 2(1)$ & $P-1$ \\
\hline$a(\AA)$ & $9.5402(10)$ & $10.488(4)$ & $11.4182(12)$ \\
\hline$b(\AA)$ & $18.245(2)$ & $17.139(7)$ & $11.9745(13)$ \\
\hline$c(\AA)$ & $24.821(3)$ & $23.142(9)$ & $21.951(2)$ \\
\hline$\alpha\left(^{\circ}\right)$ & 90.00 & 90.00 & $102.393(2)$ \\
\hline$\beta\left(^{\circ}\right)$ & 90.00 & $97.287(9)$ & 90.00 \\
\hline$\gamma\left({ }^{\circ}\right)$ & 90.00 & 90.00 & $116.379(2)$ \\
\hline Volume $\left(\AA^{3}\right)$ & $4320.4(8)$ & $4126(3)$ & $2606.2(5)$ \\
\hline$Z$ & 4 & 4 & 2 \\
\hline Density $_{\text {calc }}\left(\mathrm{Mg} / \mathrm{m}^{3}\right)$ & 1.234 & 1.110 & 1.223 \\
\hline Abs coeff $\left(\mathrm{mm}^{-1}\right)$ & 0.781 & 0.682 & 0.560 \\
\hline$F(000)$ & 1704 & 1496 & 1024 \\
\hline$\theta$ range $\left(^{\circ}\right)$ & 1.385 to 30.460 & 0.89 to 25.05 & 0.957 to 25.495 \\
\hline Data collected $(h k l)$ & $\pm 13,-26$ to $22,-34$ to 35 & -12 to $8,-20$ to $20,-27$ to 26 & $\pm 13,-14$ to $12, \pm 26$ \\
\hline Reflns collected/unique & $42689 / 13110$ & $22620 / 14539$ & $17757 / 9607$ \\
\hline $\mathrm{R}$ (int) & 0.0367 & 0.1092 & 0.0356 \\
\hline Max. and min. transmn & 0.9592 and 0.8726 & 1.0000 and 0.0705 & 0.9592 and 0.8726 \\
\hline Data / restrains / para & $13110 / 0 / 458$ & 14539 / 463 / 807 & $9607 / 7 / 700$ \\
\hline & 0.990 & 1.010 & 1.214 \\
\hline \multicolumn{4}{|l|}{ Goodness-of-fit on $F^{2}$} \\
\hline Final $R_{1}, w R_{2}[\mathrm{I}>2 \sigma(\mathrm{I})]$ & $0.0298,0.0653$ & $0.1152,0.2782$ & $0.0935,0.2287$ \\
\hline$R_{1}, w R_{2}$ (all data) & $0.0385,0.0684$ & $0.1803,0.3263$ & $0.1025,0.2395$ \\
\hline$\Delta \rho_{\max , \min } \mathrm{e} \AA^{-3}$ & 0.434 and -0.841 & 1.838 and -1.267 & 0.565 and -0.889 \\
\hline
\end{tabular}


Table S2. Crystallographic data and structure refinement for complexes $\mathbf{7 a}, 10 \mathbf{b}$ and $\mathbf{1 1}$

\begin{tabular}{|c|c|c|c|}
\hline & $7 \mathbf{a}$ & $10 b$ & 11 \\
\hline Empirical formula & $\mathrm{C}_{45} \mathrm{H}_{65} \mathrm{~N}_{3} \mathrm{OSi}_{2} \mathrm{Zn}$ & $\mathrm{C}_{41} \mathrm{H}_{52} \mathrm{~N}_{2} \mathrm{OZn}$ & $\mathrm{C}_{39} \mathrm{H}_{47} \mathrm{ClN}_{2} \mathrm{OZn}$ \\
\hline Formula weight & 785.55 & 654.21 & 660.60 \\
\hline Temp (K) & 293(2) & $140(2)$ & $293(2)$ \\
\hline Crystal size (mm) & $0.276 \times 0.203 \times 0.076$ & $0.250 \times 0.200 \times 0.150$ & $0.330 \times 0.280 \times 0.2306$ \\
\hline Crystal system & Monoclinic & Orthorhombic & Orthorhombic \\
\hline Space group & $P 2(1)$ & $P 2(1) 2(1) 2(1)$ & $P 2(1) 2(1) 2(1)$ \\
\hline$a(\AA)$ & $15.123(2)$ & $8.3865(9)$ & $12.2375(13)$ \\
\hline$b(\AA)$ & $10.8882(14)$ & $17.5334(19)$ & $16.5783(18)$ \\
\hline$c(\AA)$ & $16.463(2)$ & $23.826(3)$ & $17.4078(18)$ \\
\hline$\alpha\left(^{\circ}\right)$ & 90.00 & 90.00 & 90.00 \\
\hline$\beta\left(^{\circ}\right)$ & $111.496(3)$ & 90.00 & 90.00 \\
\hline$\gamma\left({ }^{\circ}\right)$ & 90.00 & 90.00 & 90.00 \\
\hline Volume $\left(\AA^{3}\right)$ & $2522.2(6)$ & $3503.5(7)$ & $3531.6(6)$ \\
\hline$Z$ & 2 & 4 & 4 \\
\hline Density $_{\text {calc }}\left(\mathrm{Mg} / \mathrm{m}^{3}\right)$ & 1.034 & 1.240 & 1.242 \\
\hline Abs coeff $\left(\mathrm{mm}^{-1}\right)$ & 0.565 & 0.735 & 0.803 \\
\hline$F(000)$ & 844 & 1400 & 1400 \\
\hline$\theta$ range $\left(^{\circ}\right)$ & 1.45 to 25.50 & 1.442 to 30.597 & 1.696 to 30.541 \\
\hline Data collected $(h k l)$ & -18 to $17,-13$ to 10,0 to 19 & -12 to $11, \pm 25,-34$ to 28 & $\pm 17, \pm 23,-16$ to 24 \\
\hline Reflns collected/unique & $7582 / 7582$ & $34764 / 10746$ & $35008 / 10798$ \\
\hline $\mathrm{R}$ (int) & 0.0000 & 0.0458 & 0.0440 \\
\hline Max. and min. transmn & 1.00000 and 0.12976 & 0.7461 and 0.6622 & 0.7461 and 0.6648 \\
\hline Data / restrains / para & $7582 / 1 / 479$ & $10746 / 0 / 412$ & 10798 / 0 / 402 \\
\hline & 0.937 & 0.992 & 0.974 \\
\hline \multicolumn{4}{|l|}{ Goodness-of-fit on $F^{2}$} \\
\hline Final $R_{1}, w R_{2}[\mathrm{I}>2 \sigma(\mathrm{I})]$ & $0.0622,0.1359$ & $0.0346,0.0769$ & $0.0395,0.0890$ \\
\hline$R_{1}, w R_{2}$ (all data) & $0.0930,0.1487$ & $0.0528,0.0834$ & $0.0700,0.1019$ \\
\hline$\Delta \rho_{\max , \min / \mathrm{e} \AA^{-3}}$ & 0.736 and -0.353 & 0.260 and -0.247 & 0.226 and -0.354 \\
\hline
\end{tabular}




\section{3. ${ }^{1}$ H NMR and ${ }^{13}$ C NMR Spectra of Complexes 1-11}

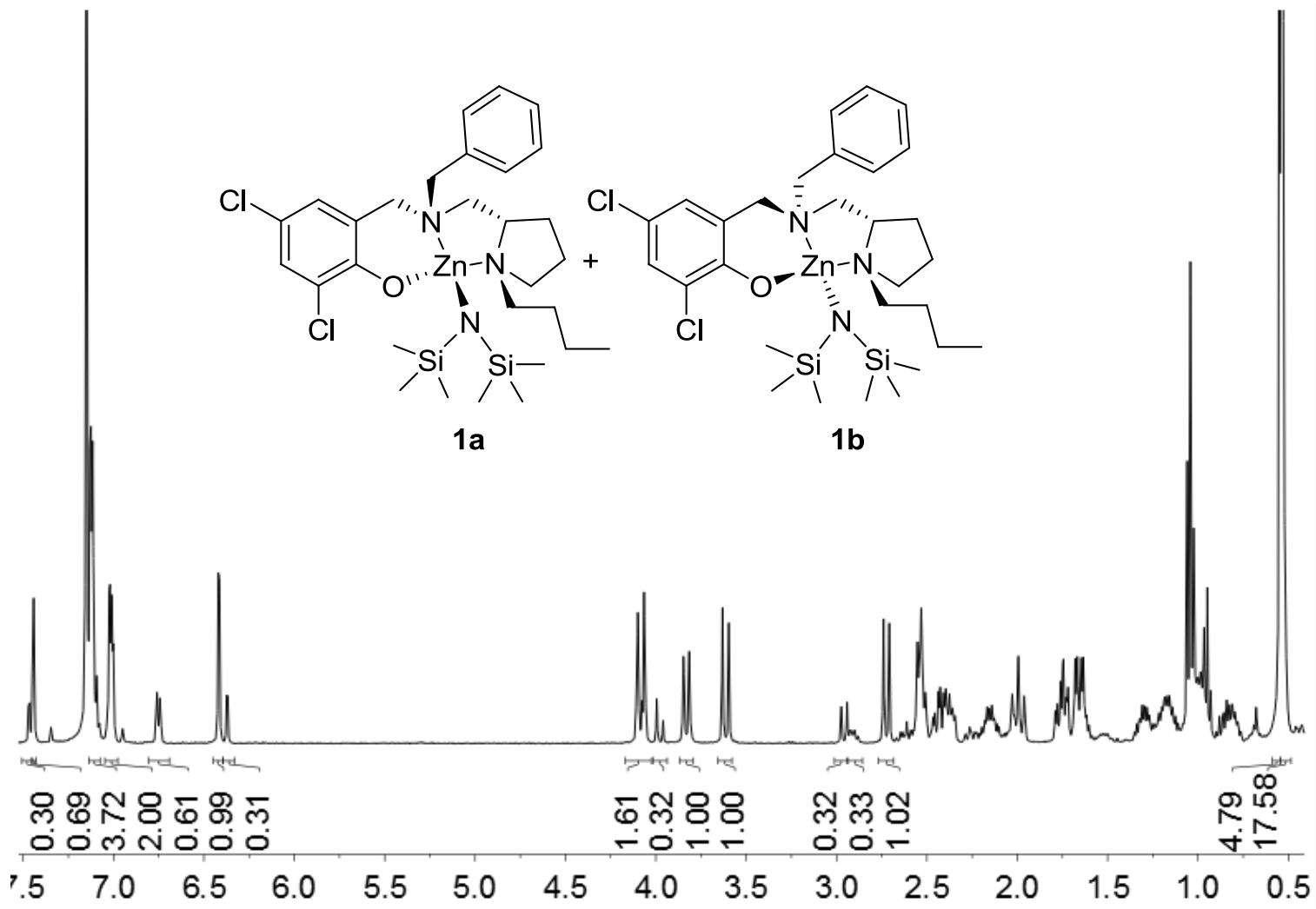

Figure S1. ${ }^{1} \mathrm{H}$ NMR spectrum $\left(\mathrm{C}_{6} \mathrm{D}_{6}, 400 \mathrm{MHz}, 20^{\circ} \mathrm{C}\right)$ of $\left[(S)-\mathrm{L}^{1}\right] \mathrm{ZnN}\left(\mathrm{SiMe}_{3}\right)_{2}(\mathbf{1})$.

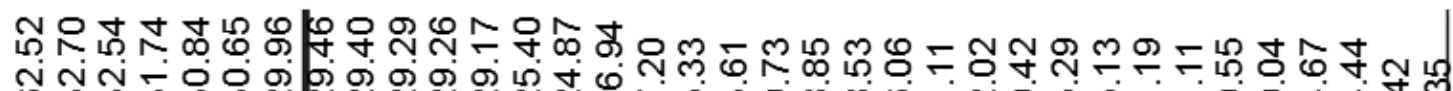
ஸั

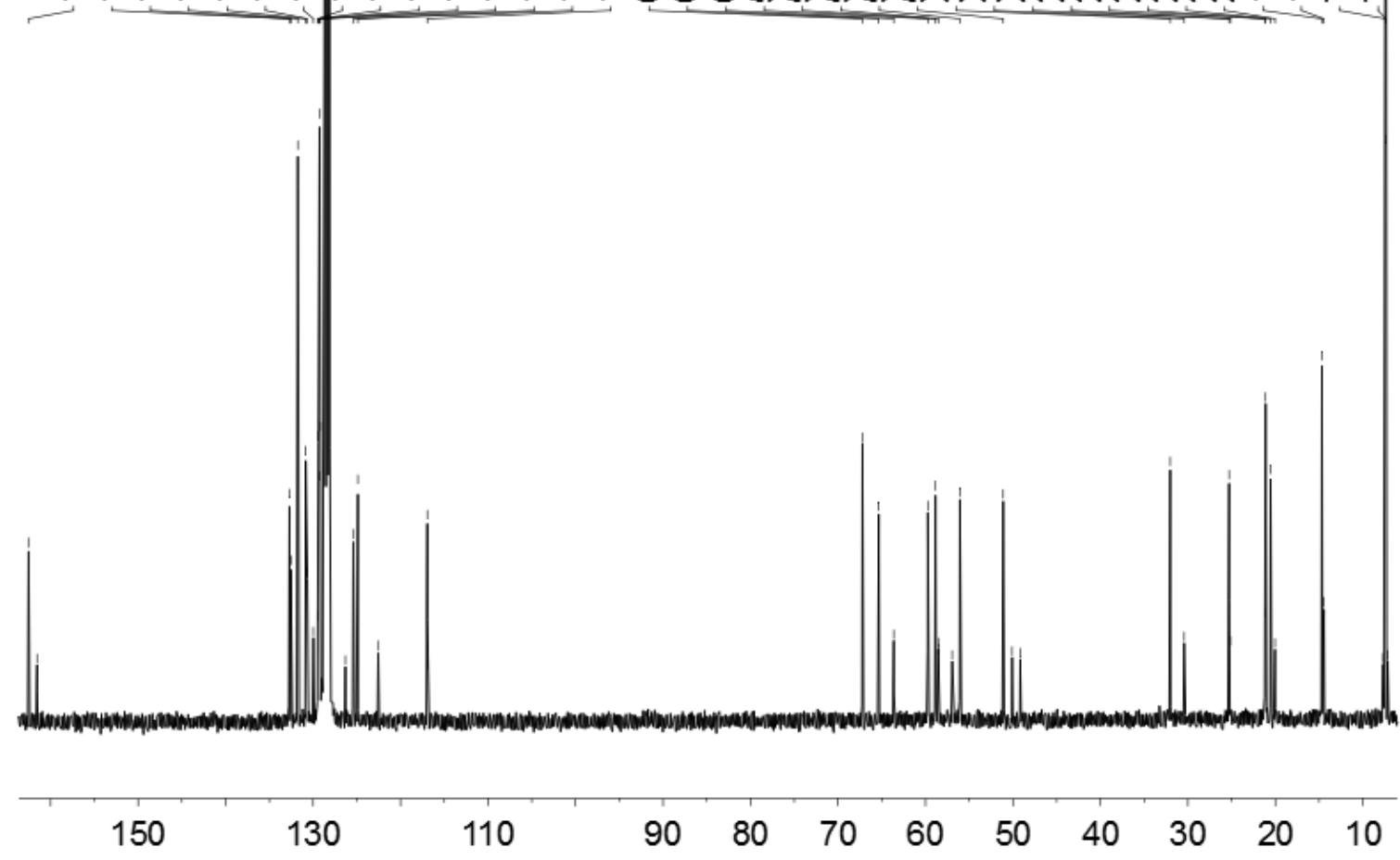

Figure S2. ${ }^{13} \mathrm{C}\left\{{ }^{1} \mathrm{H}\right\}$ NMR spectrum $\left(\mathrm{C}_{6} \mathrm{D}_{6}, 400 \mathrm{MHz}, 20{ }^{\circ} \mathrm{C}\right)$ of $\left[(S)-\mathrm{L}^{1}\right] \mathrm{ZnN}\left(\mathrm{SiMe}_{3}\right)_{2}(\mathbf{1})$. 


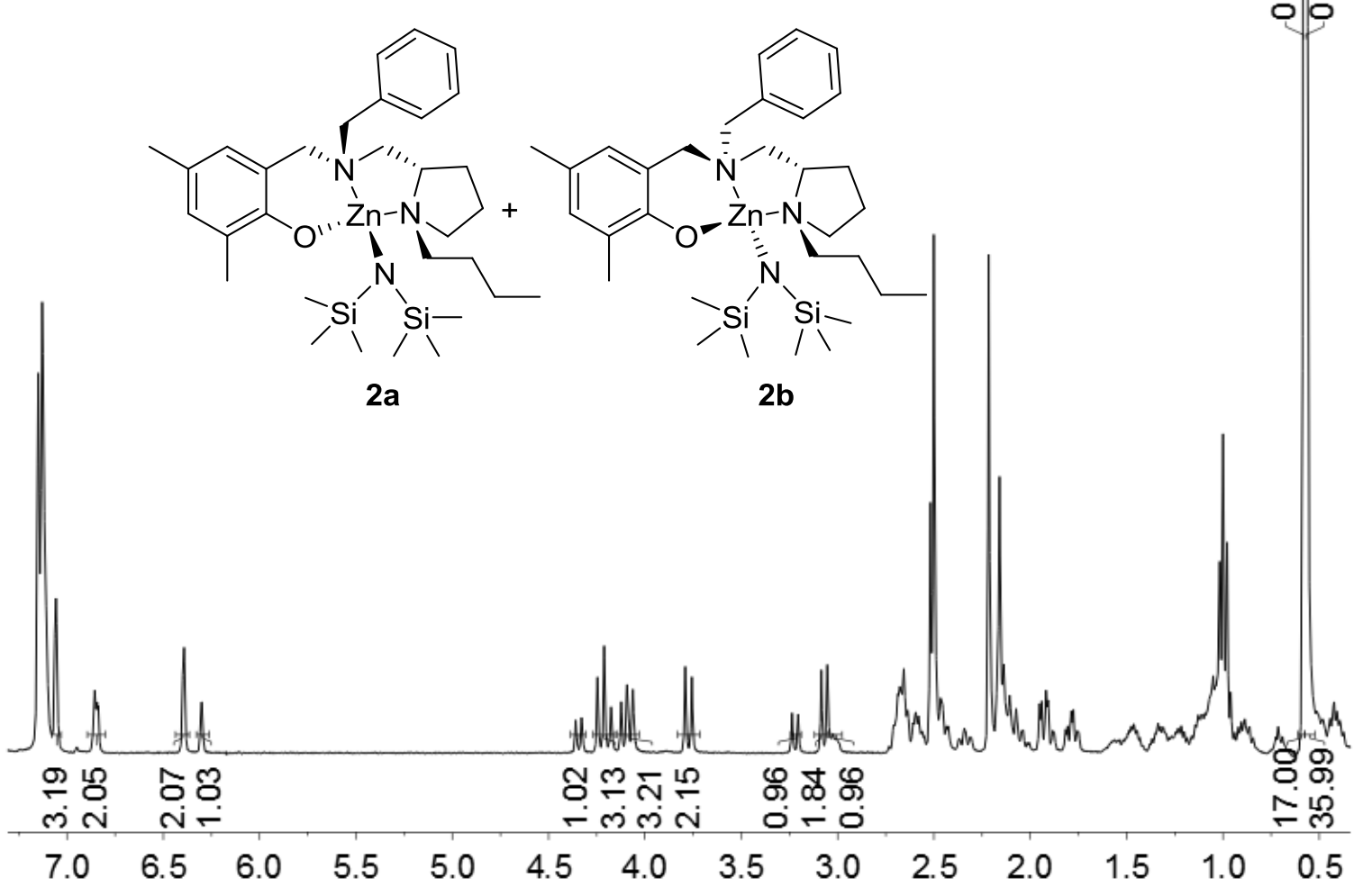

Figure S3. ${ }^{1} \mathrm{H}$ NMR spectrum $\left(\mathrm{C}_{6} \mathrm{D}_{6}, 400 \mathrm{MHz}, 20{ }^{\circ} \mathrm{C}\right)$ of $\left[(S)-\mathrm{L}^{2}\right] \mathrm{ZnN}\left(\mathrm{SiMe}_{3}\right)_{2}(2)$.

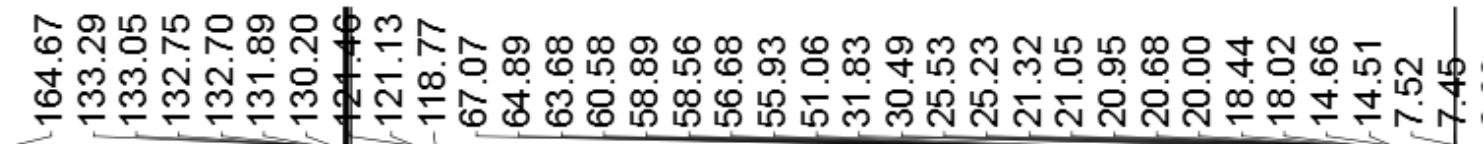

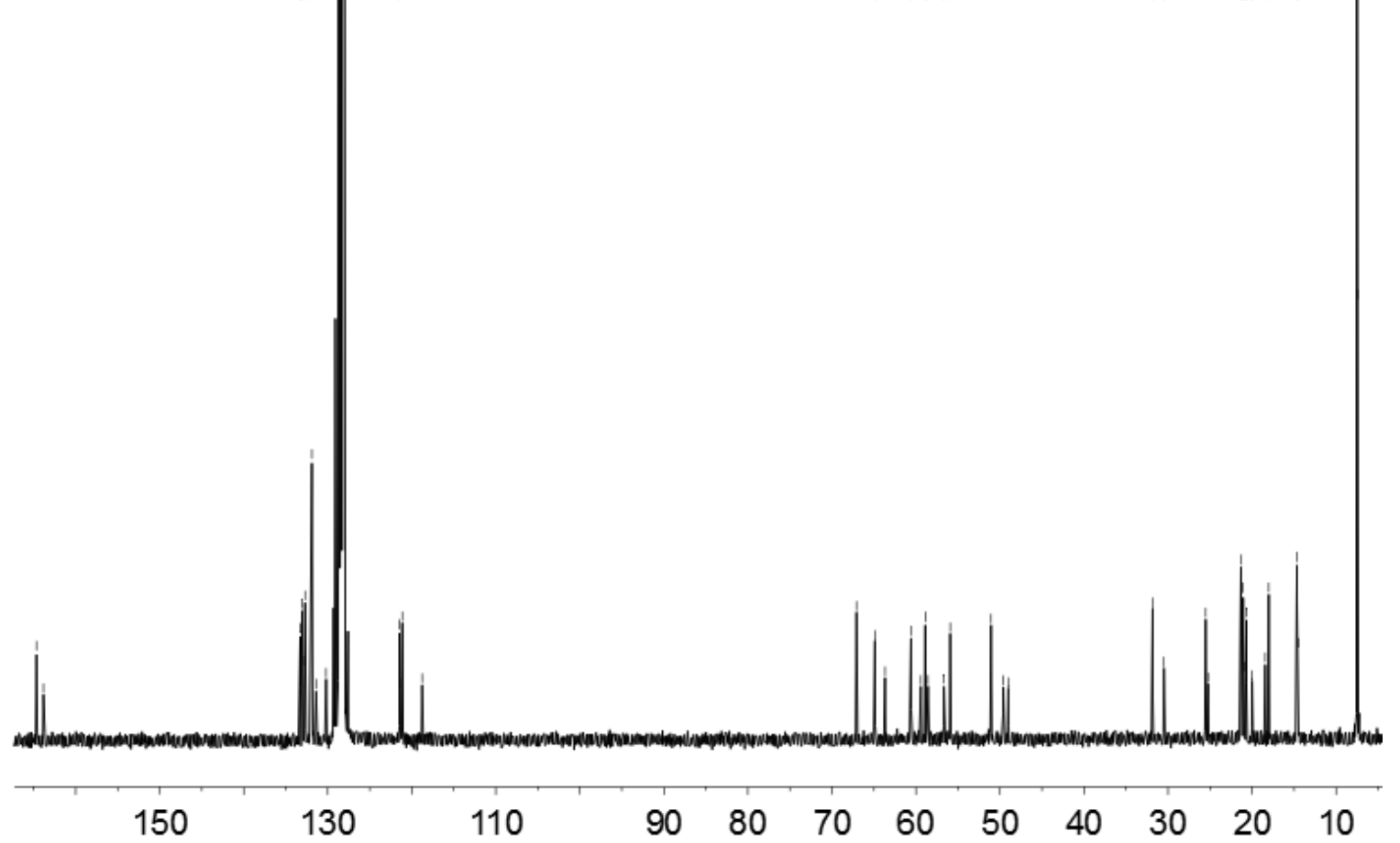

Figure S4. ${ }^{13} \mathrm{C}\left\{{ }^{1} \mathrm{H}\right\}$ NMR spectrum $\left(\mathrm{C}_{6} \mathrm{D}_{6}, 400 \mathrm{MHz}, 20{ }^{\circ} \mathrm{C}\right)$ of $\left[(S)-\mathrm{L}^{2}\right] \mathrm{ZnN}\left(\mathrm{SiMe}_{3}\right)_{2}(2)$. 


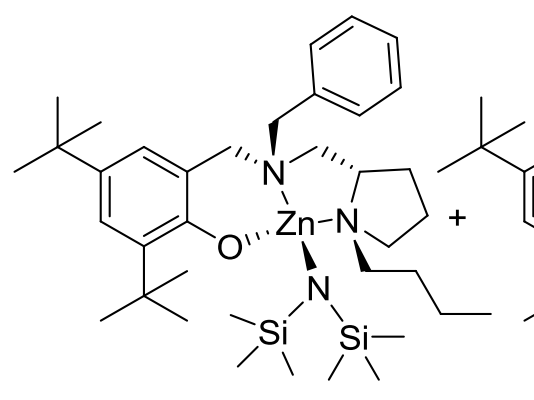

$3 a$

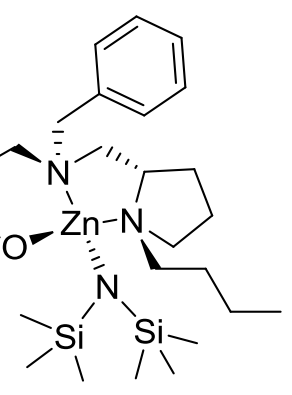

$3 \mathbf{b}$

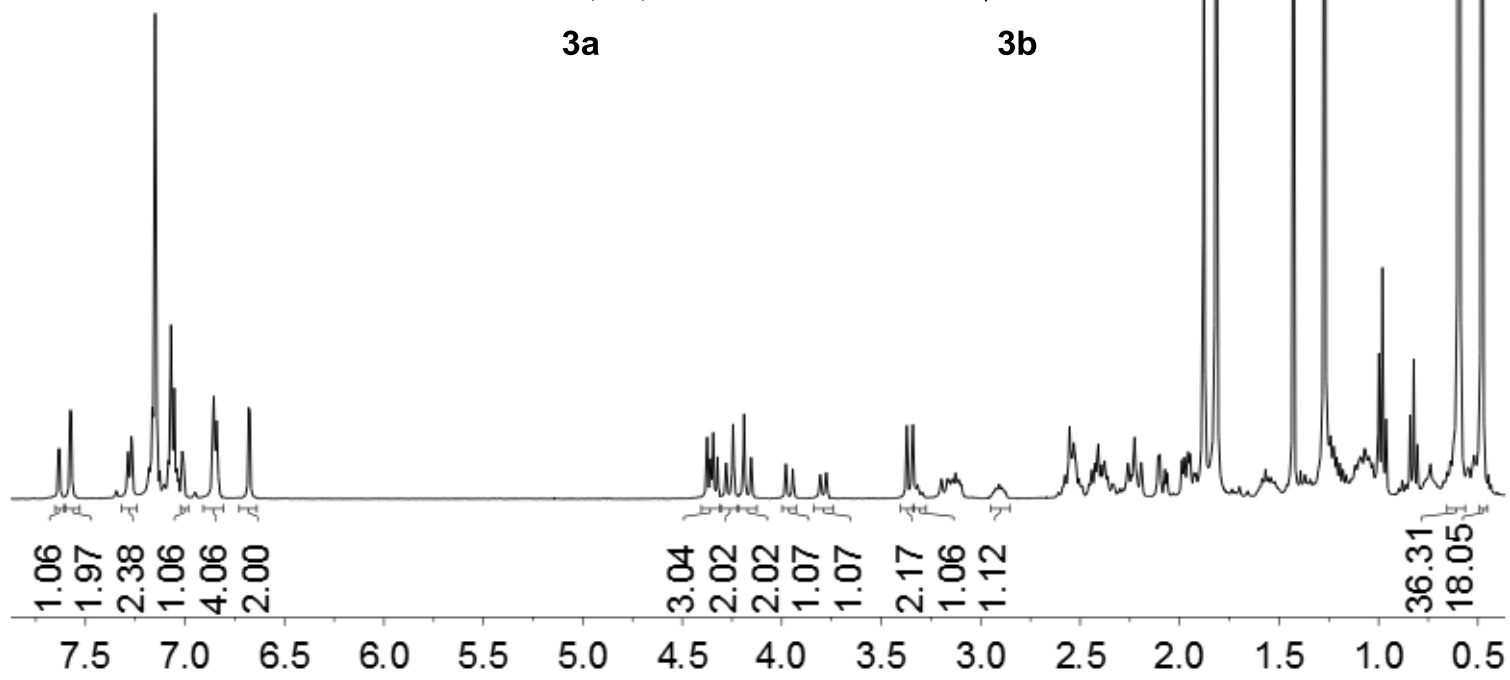

Figure S5. ${ }^{1} \mathrm{H}$ NMR spectrum $\left(\mathrm{C}_{6} \mathrm{D}_{6}, 400 \mathrm{MHz}, 20{ }^{\circ} \mathrm{C}\right)$ of $\left[(S)-\mathrm{L}^{3}\right] \mathrm{ZnN}\left(\mathrm{SiMe}_{3}\right)_{2}(3)$.

L m

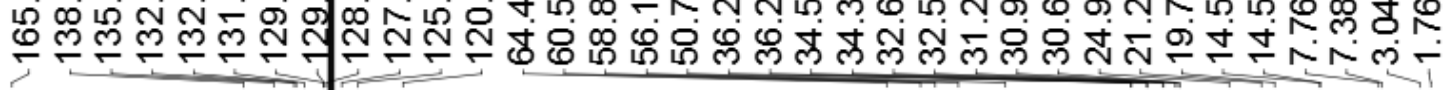

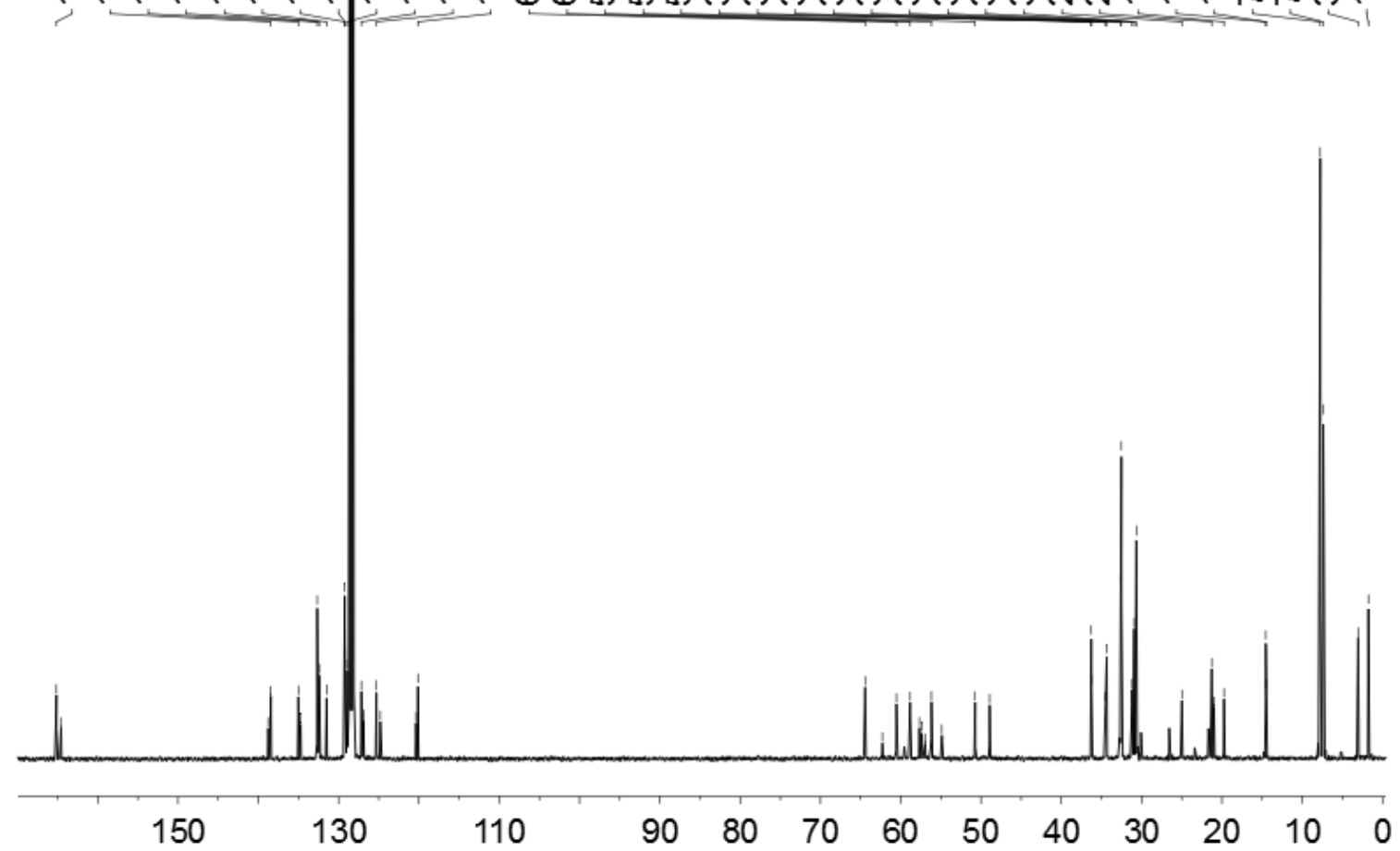

Figure S6. ${ }^{13} \mathrm{C}\left\{{ }^{1} \mathrm{H}\right\}$ NMR spectrum $\left(\mathrm{C}_{6} \mathrm{D}_{6}, 400 \mathrm{MHz}, 20{ }^{\circ} \mathrm{C}\right)$ of $\left[(S)-\mathrm{L}^{3}\right] \mathrm{ZnN}\left(\mathrm{SiMe}_{3}\right)_{2}(3)$. 


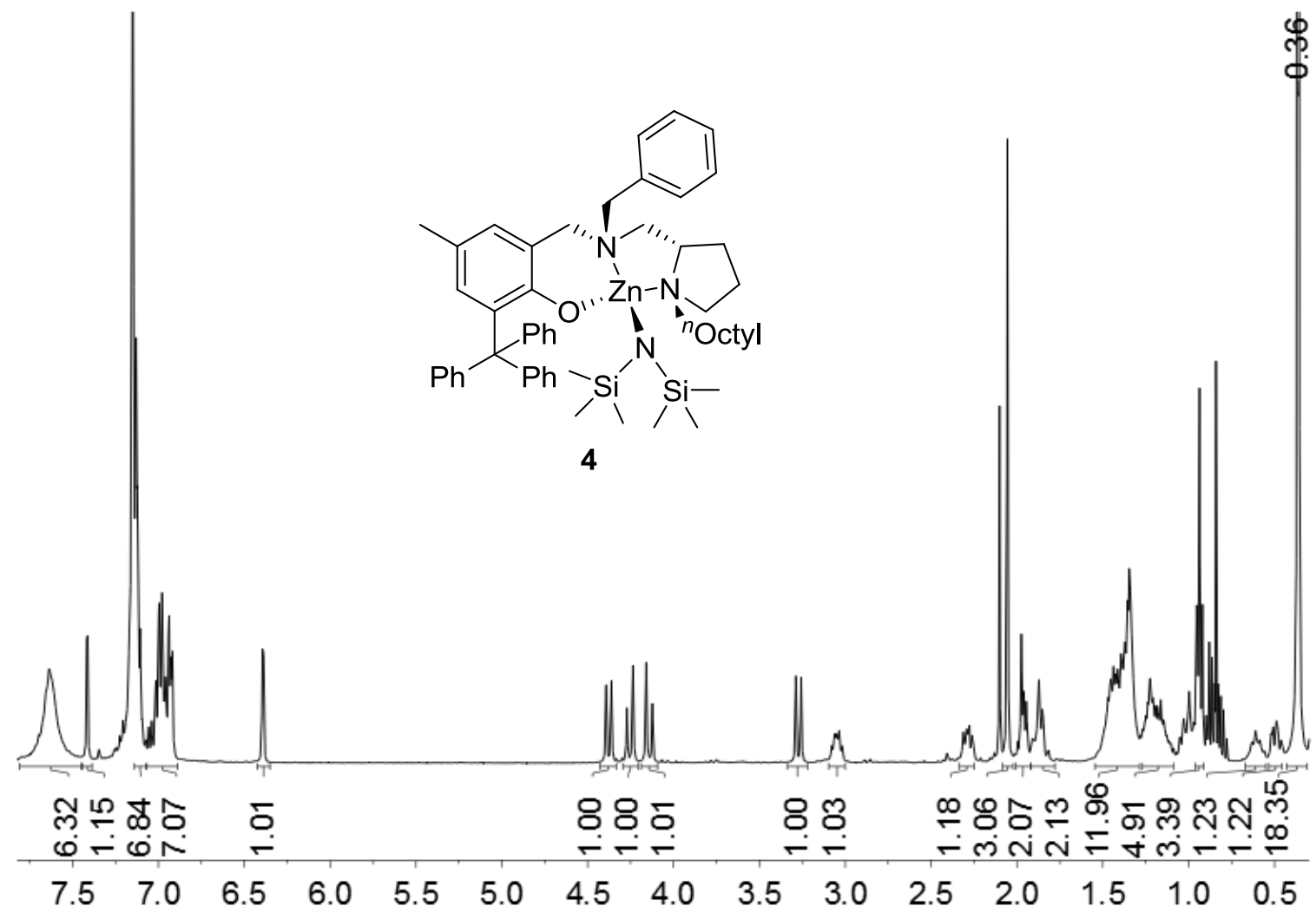

Figure S7. ${ }^{1} \mathrm{H}$ NMR spectrum $\left(\mathrm{C}_{6} \mathrm{D}_{6}, 400 \mathrm{MHz}, 20{ }^{\circ} \mathrm{C}\right)$ of $\left[(S)-\mathrm{L}^{4}\right] \mathrm{ZnN}\left(\mathrm{SiMe}_{3}\right)_{2}$ (4).

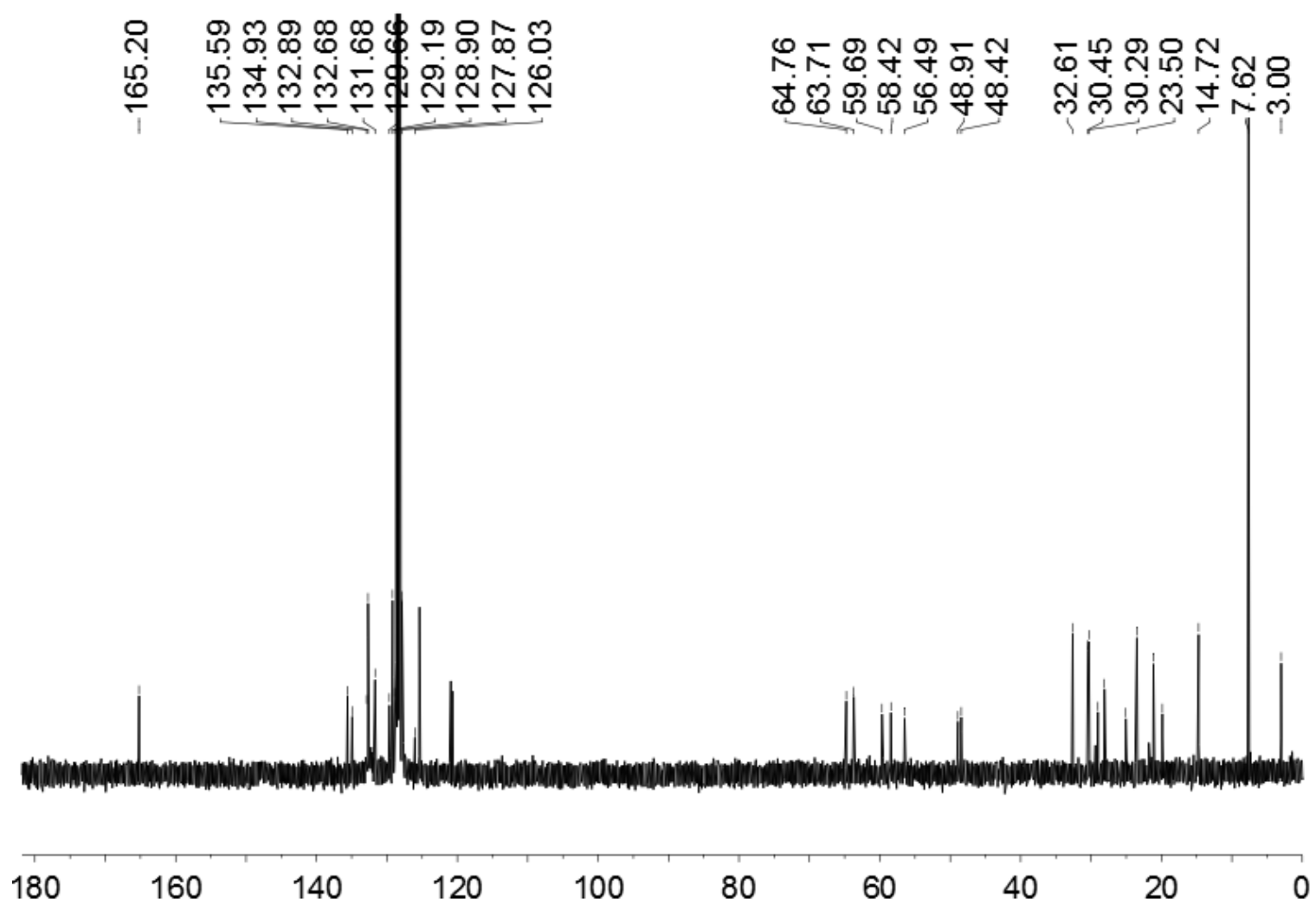

Figure S8. ${ }^{13} \mathrm{C}\left\{{ }^{1} \mathrm{H}\right\}$ NMR spectrum $\left(\mathrm{C}_{6} \mathrm{D}_{6}, 400 \mathrm{MHz}, 20{ }^{\circ} \mathrm{C}\right)$ of $\left[(S)-\mathrm{L}^{4}\right] \mathrm{ZnN}\left(\mathrm{SiMe}_{3}\right)_{2}(4)$. 


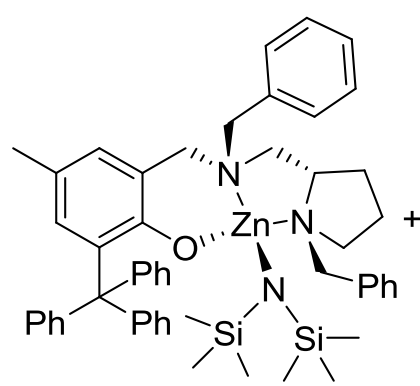

$5 a$

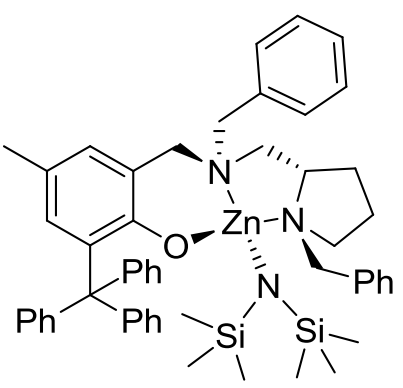

5b

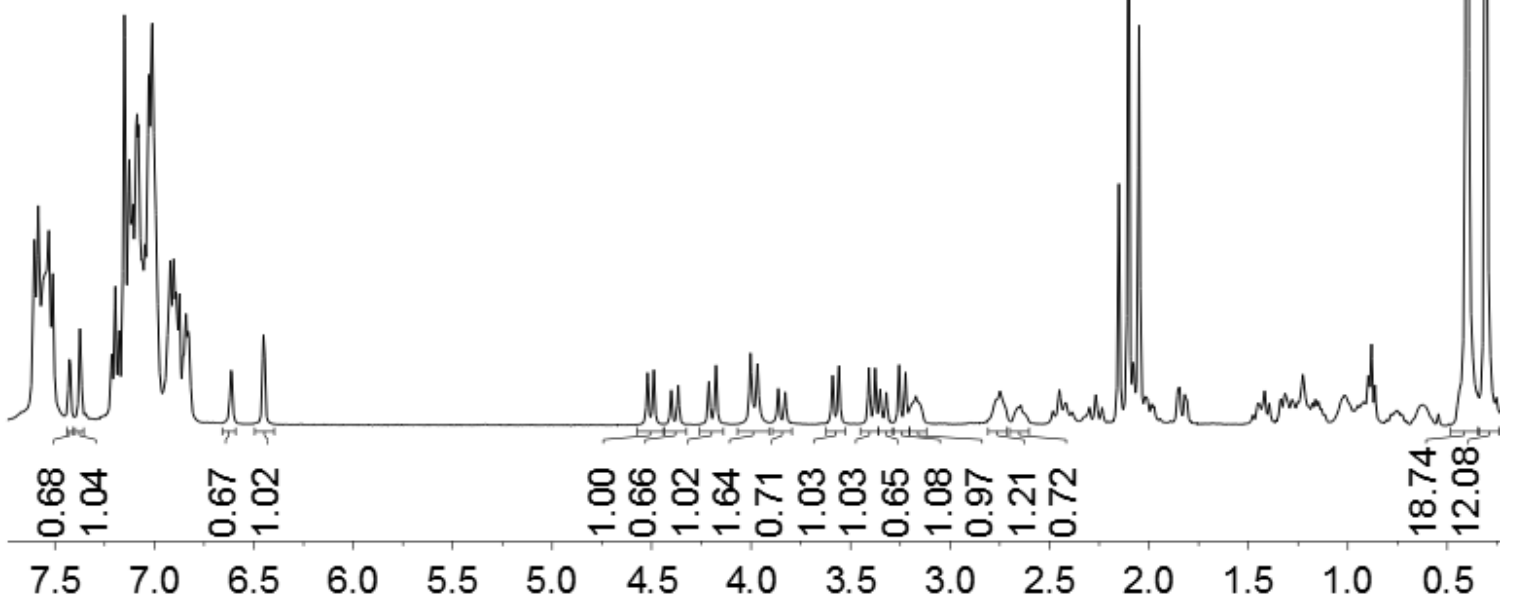

Figure S9. ${ }^{1} \mathrm{H}$ NMR spectrum $\left(\mathrm{C}_{6} \mathrm{D}_{6}, 400 \mathrm{MHz}, 20{ }^{\circ} \mathrm{C}\right)$ of $\left[(S)-\mathrm{L}^{5}\right] \mathrm{ZnN}\left(\mathrm{SiMe}_{3}\right)_{2}(\mathbf{5})$.

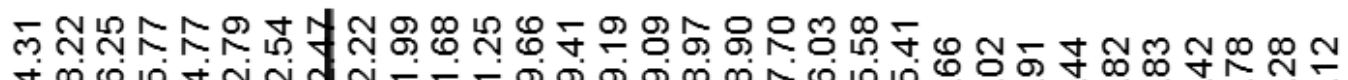

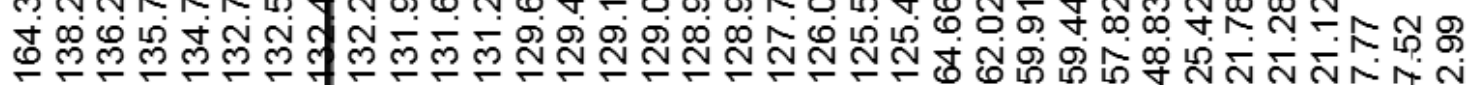

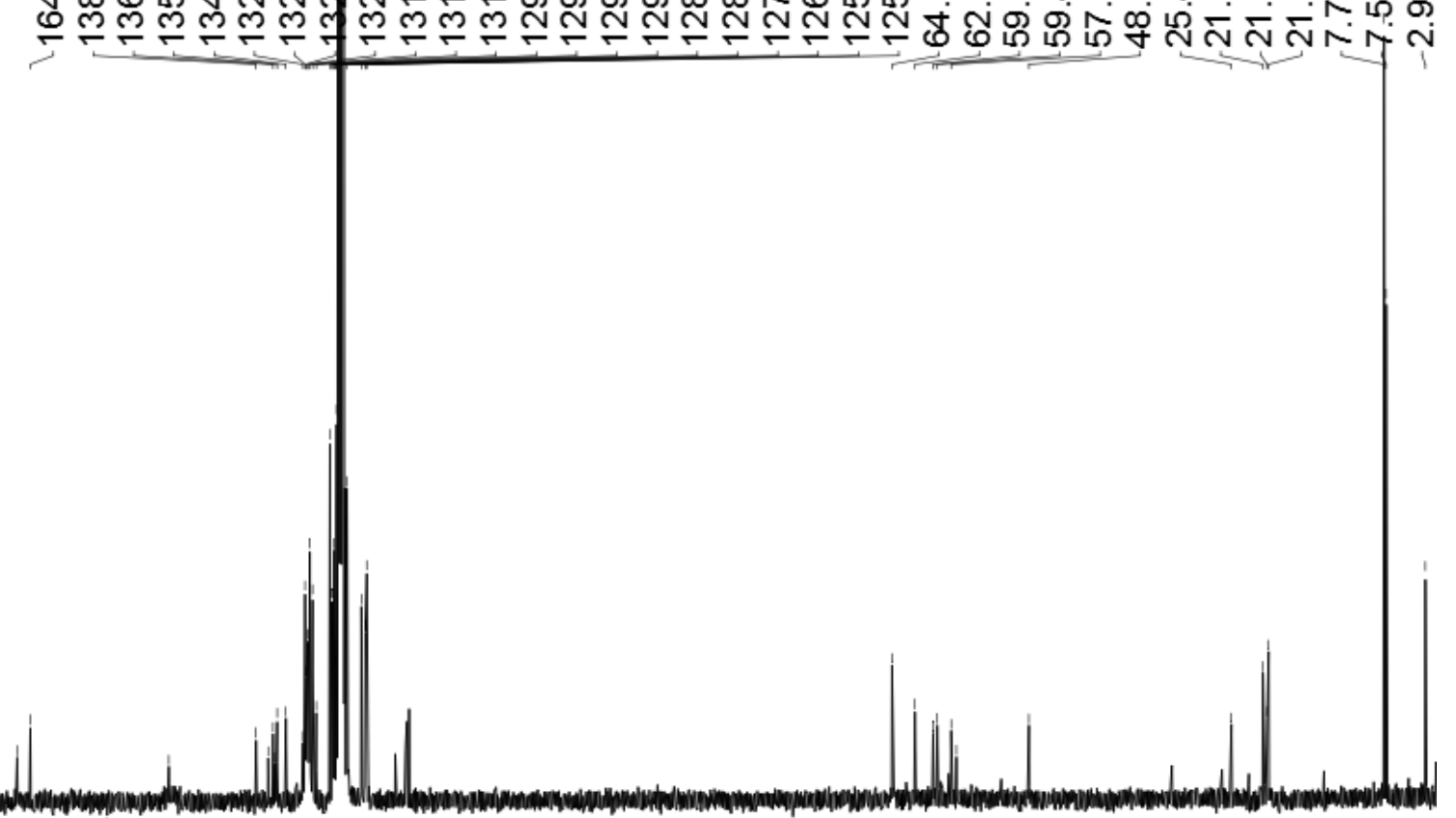

150

130

$\begin{array}{lllllllll}90 & 80 & 70 & 60 & 50 & 40 & 30 & 20 & 10\end{array}$

Figure S10. ${ }^{13} \mathrm{C}\left\{{ }^{1} \mathrm{H}\right\}$ NMR spectrum $\left(\mathrm{C}_{6} \mathrm{D}_{6}, 400 \mathrm{MHz}, 20{ }^{\circ} \mathrm{C}\right)$ of $\left[(S)-\mathrm{L}^{5}\right] \mathrm{ZnN}\left(\mathrm{SiMe}_{3}\right)_{2}(\mathbf{5})$. 


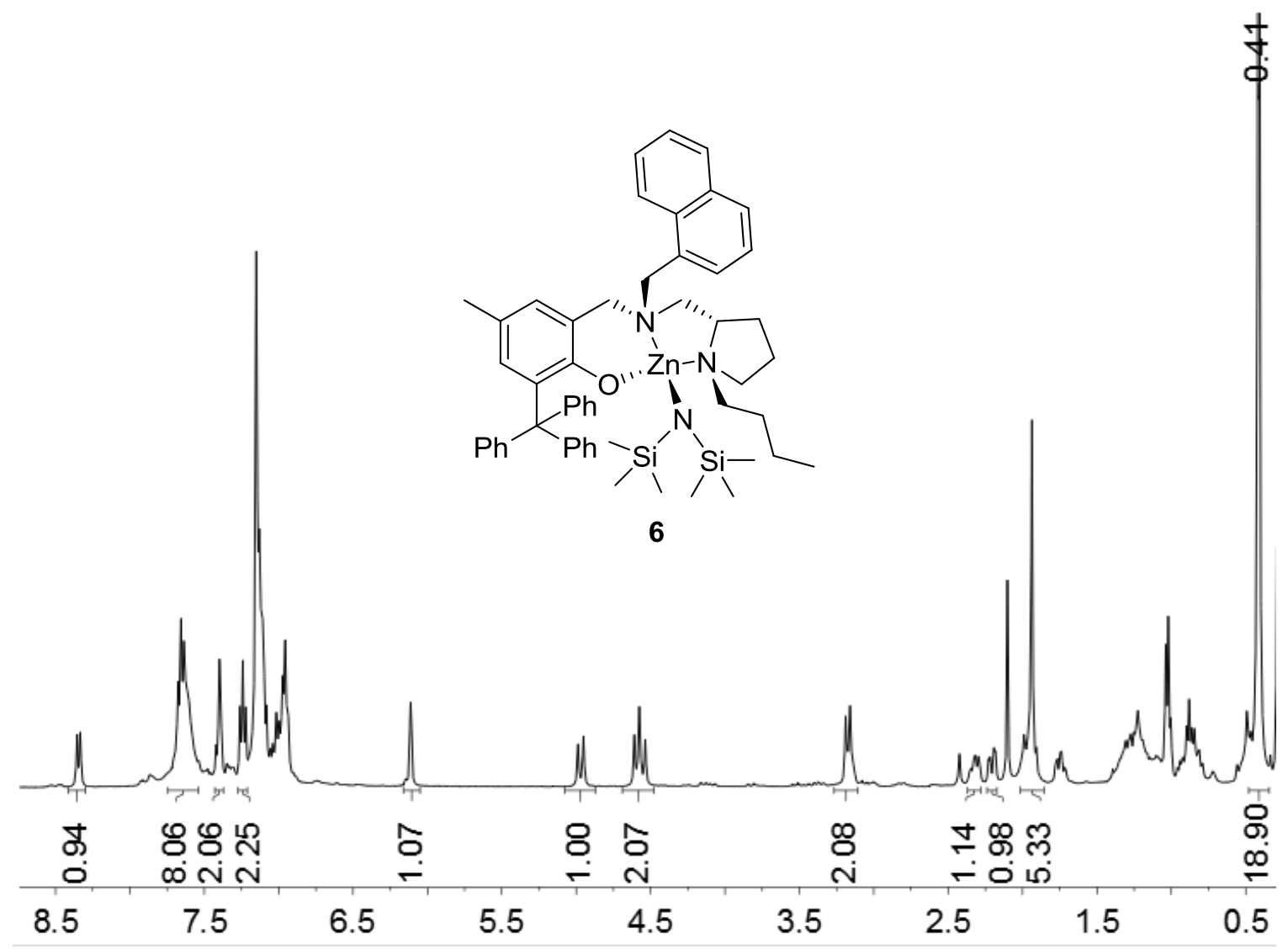

Figure S11. ${ }^{1} \mathrm{H}$ NMR spectrum $\left(\mathrm{C}_{6} \mathrm{D}_{6}, 400 \mathrm{MHz}, 20{ }^{\circ} \mathrm{C}\right)$ of $\left[(S)-\mathrm{L}^{6}\right] \mathrm{ZnN}\left(\mathrm{SiMe}_{3}\right)_{2}(6)$.

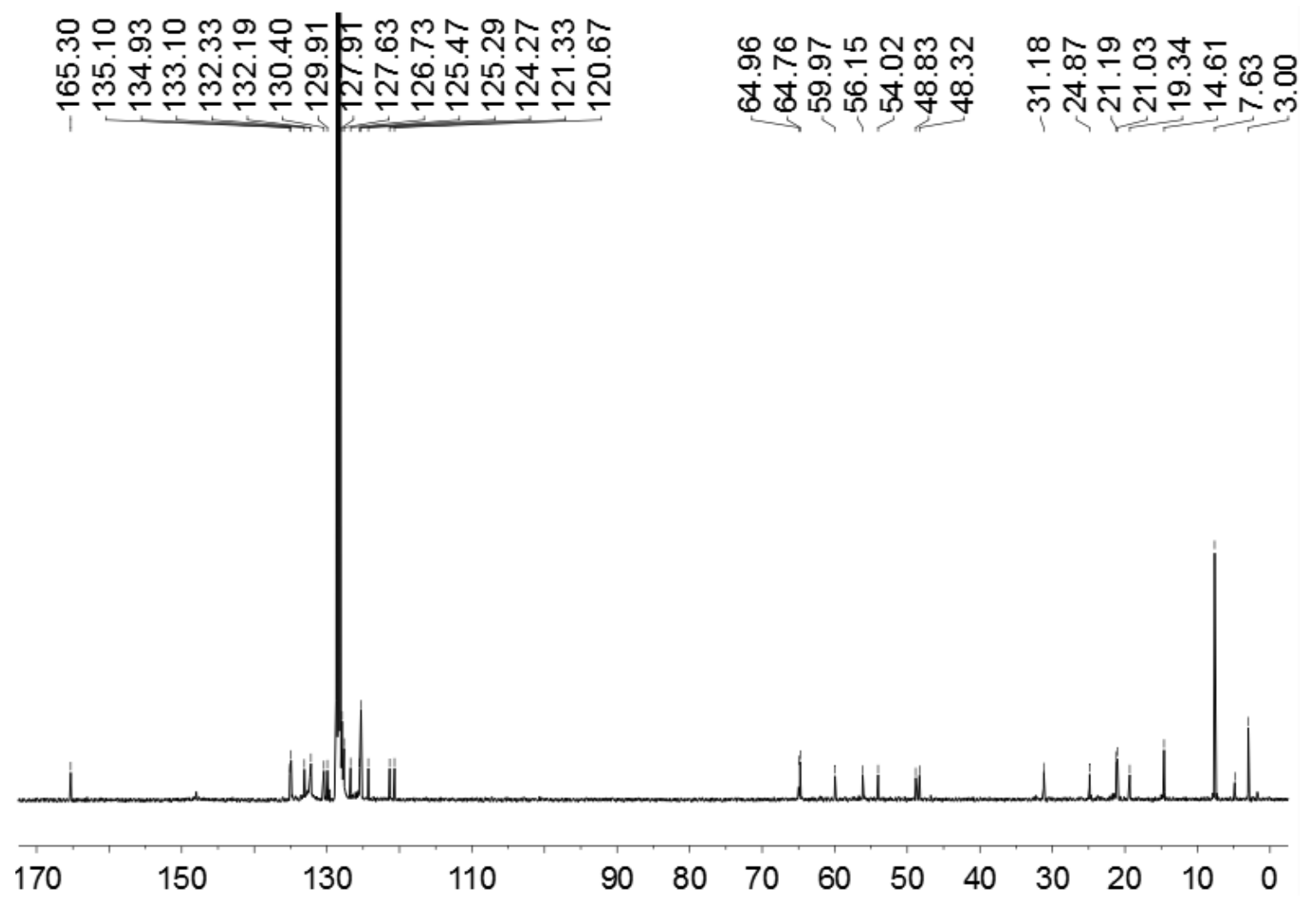

Figure S12. ${ }^{13} \mathrm{C}\left\{{ }^{1} \mathrm{H}\right\}$ NMR spectrum $\left(\mathrm{C}_{6} \mathrm{D}_{6}, 400 \mathrm{MHz}, 20{ }^{\circ} \mathrm{C}\right)$ of $\left[(S)-\mathrm{L}^{6}\right] \mathrm{ZnN}\left(\mathrm{SiMe}_{3}\right)_{2}(6)$. 


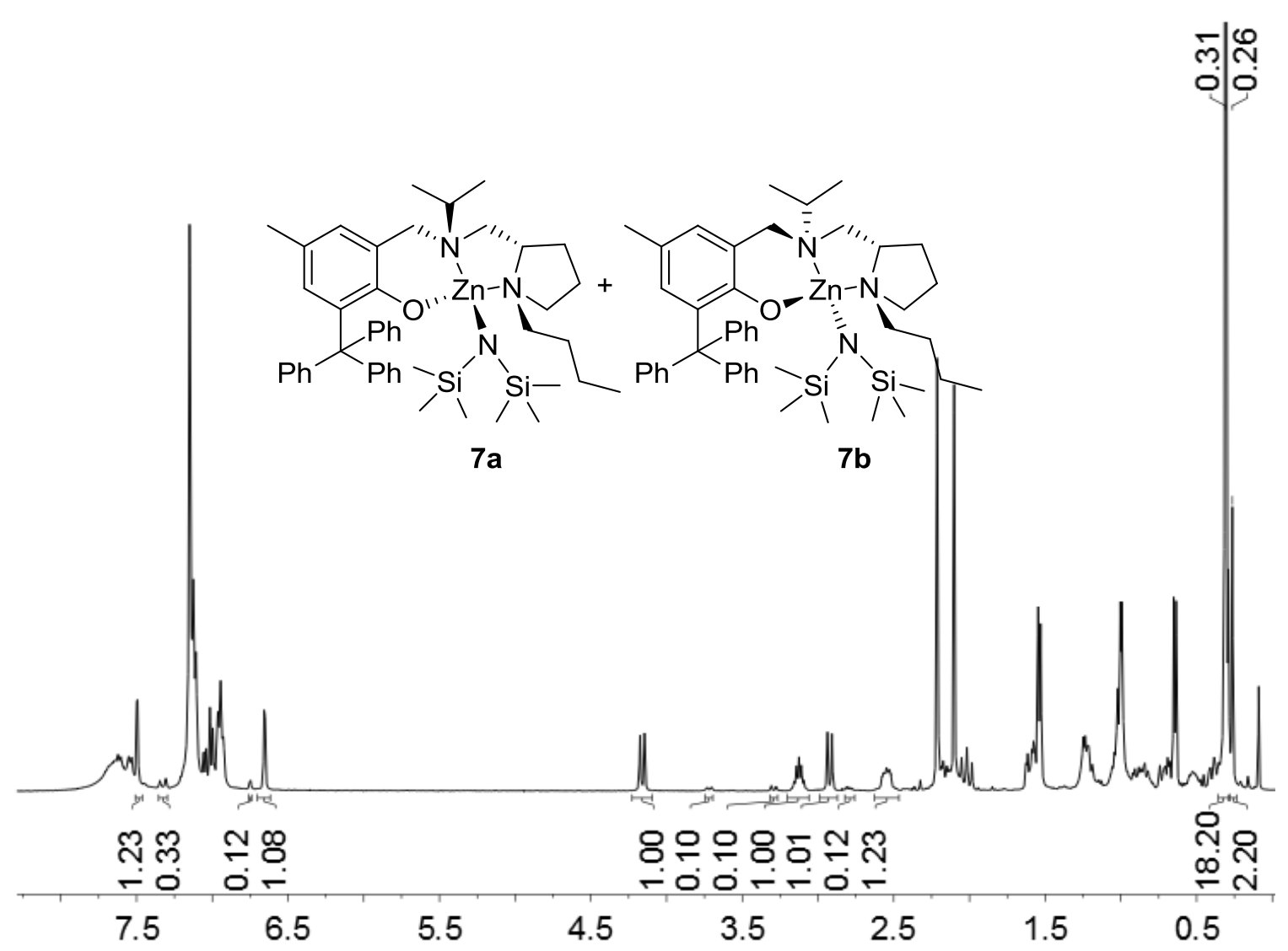

Figure S13. ${ }^{1} \mathrm{H}$ NMR spectrum $\left(\mathrm{C}_{6} \mathrm{D}_{6}, 400 \mathrm{MHz}, 20{ }^{\circ} \mathrm{C}\right)$ of $\left[(S)-\mathrm{L}^{7}\right] \mathrm{ZnN}\left(\mathrm{SiMe}_{3}\right)_{2}(7)$.

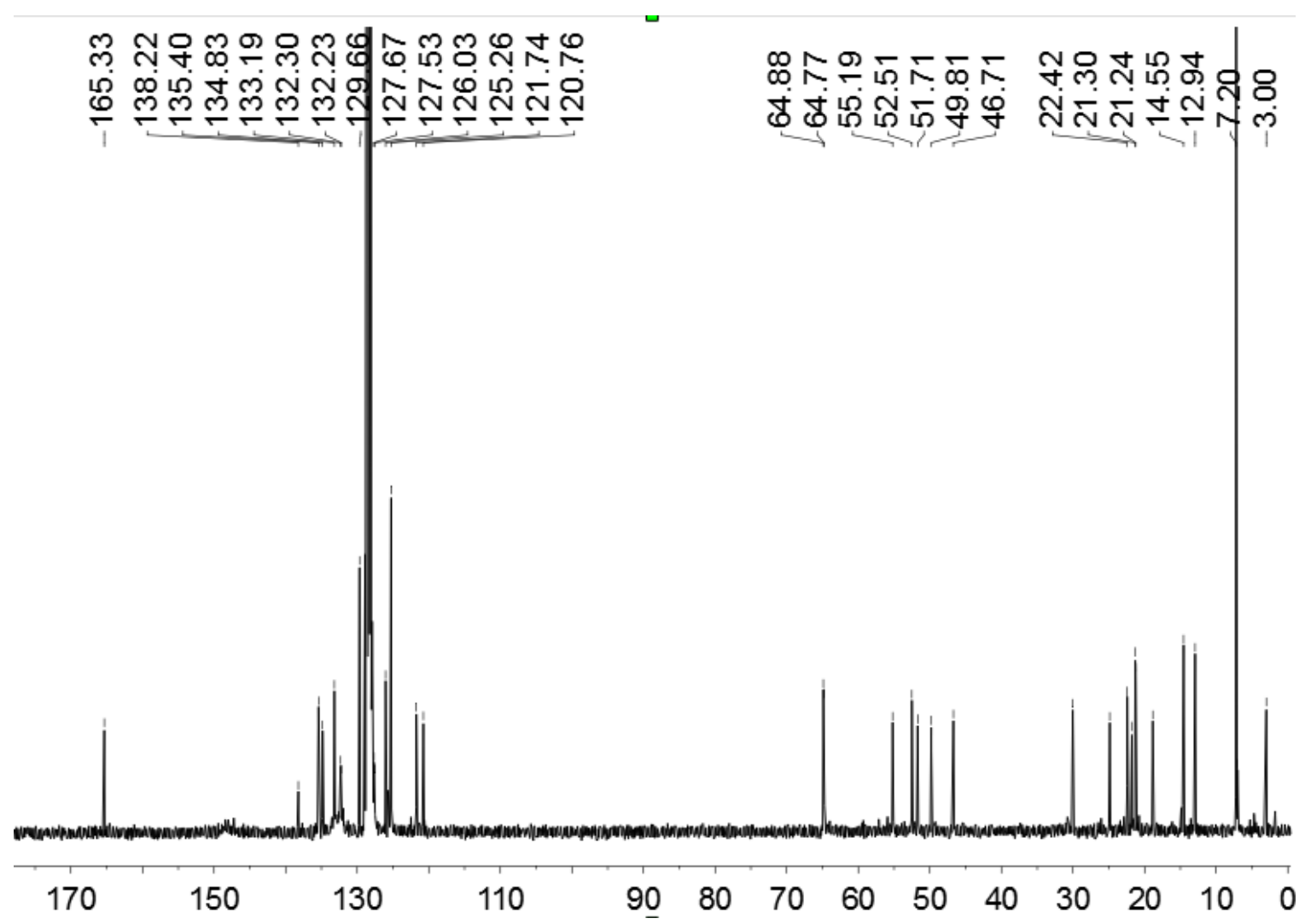

Figure S14. ${ }^{13} \mathrm{C}\left\{{ }^{1} \mathrm{H}\right\}$ NMR spectrum $\left(\mathrm{C}_{6} \mathrm{D}_{6}, 400 \mathrm{MHz}, 20{ }^{\circ} \mathrm{C}\right)$ of $\left[(S)-\mathrm{L}^{5}\right] \mathrm{ZnN}\left(\mathrm{SiMe}_{3}\right)_{2}(7)$. 


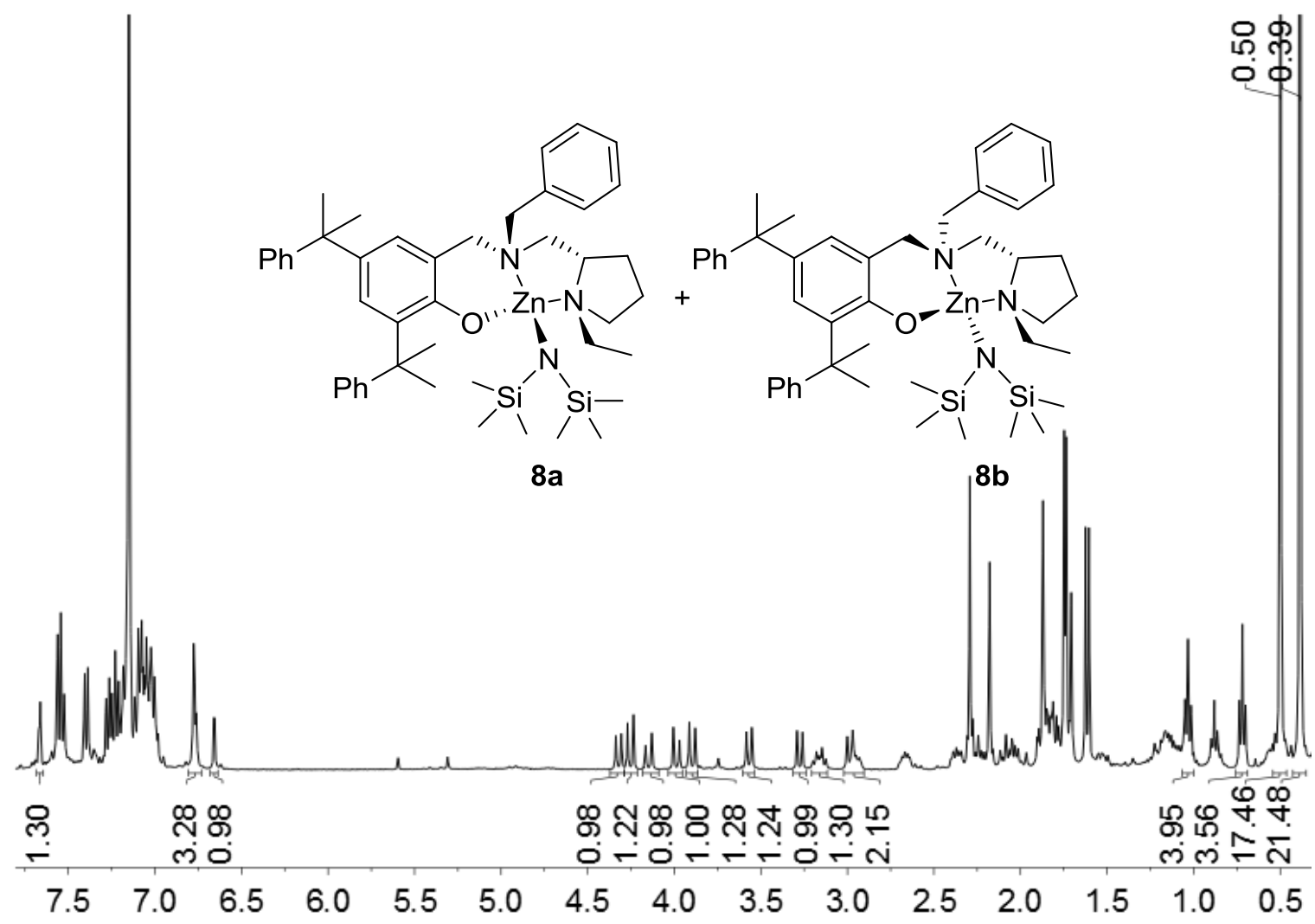

Figure S15. ${ }^{1} \mathrm{H}$ NMR spectrum $\left(\mathrm{C}_{6} \mathrm{D}_{6}, 400 \mathrm{MHz}, 20{ }^{\circ} \mathrm{C}\right)$ of $\left[(S)-\mathrm{L}^{8}\right] \mathrm{ZnN}\left(\mathrm{SiMe}_{3}\right)_{2}(8)$.

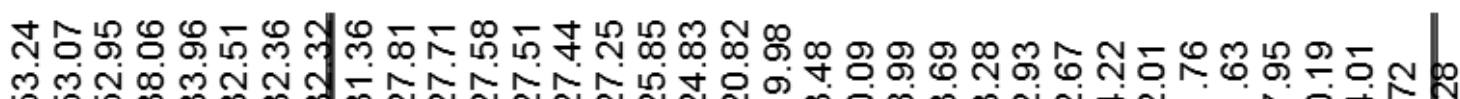

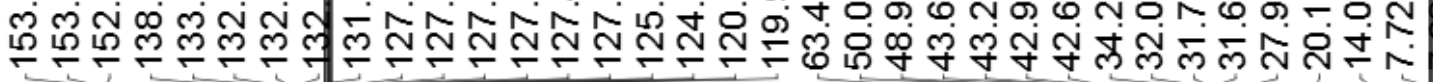

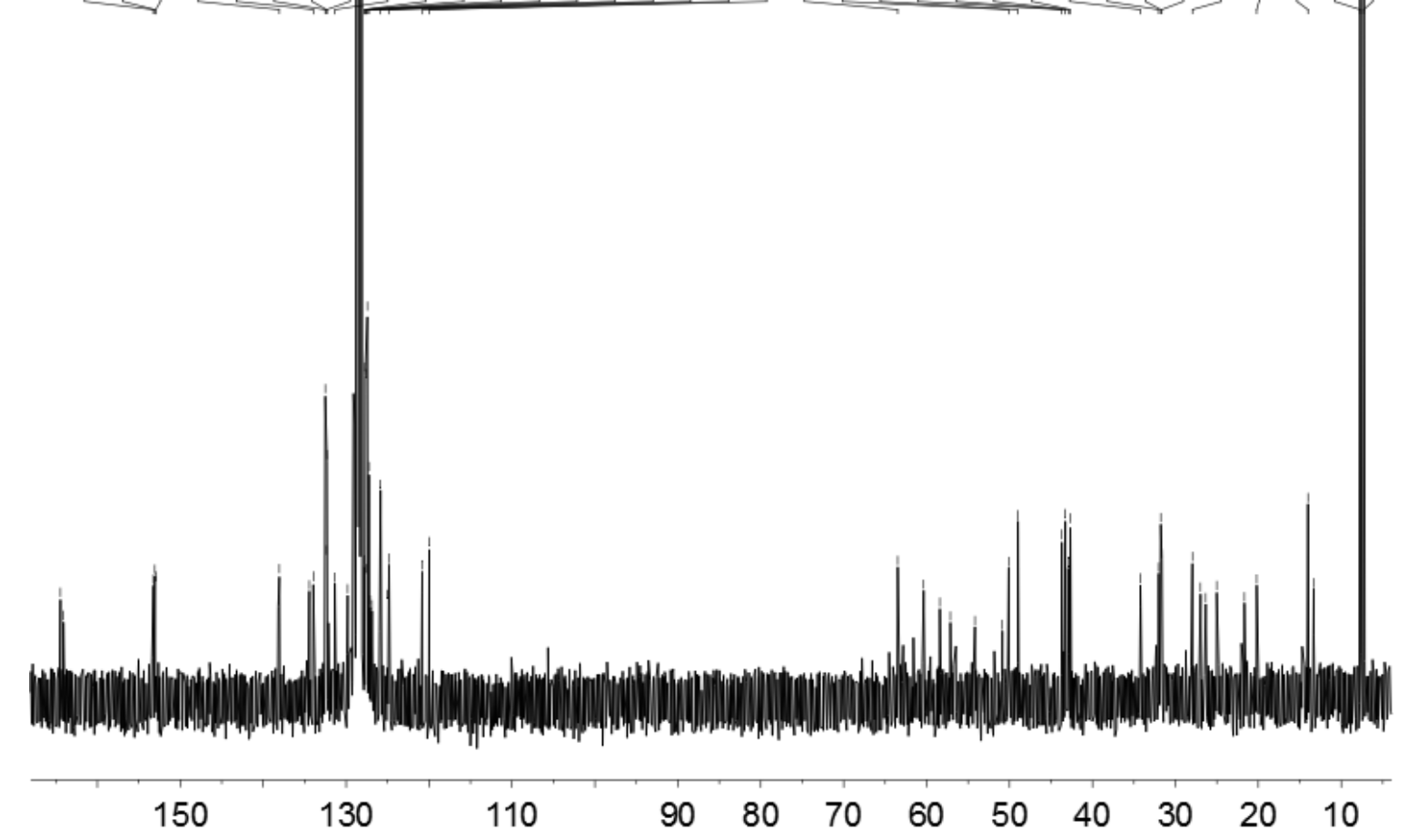

Figure S16. ${ }^{13} \mathrm{C}\left\{{ }^{1} \mathrm{H}\right\}$ NMR spectrum $\left(\mathrm{C}_{6} \mathrm{D}_{6}, 400 \mathrm{MHz}, 20{ }^{\circ} \mathrm{C}\right)$ of $\left[(S)-\mathrm{L}^{8}\right] \mathrm{ZnN}\left(\mathrm{SiMe}_{3}\right)_{2}(\mathbf{8})$. 


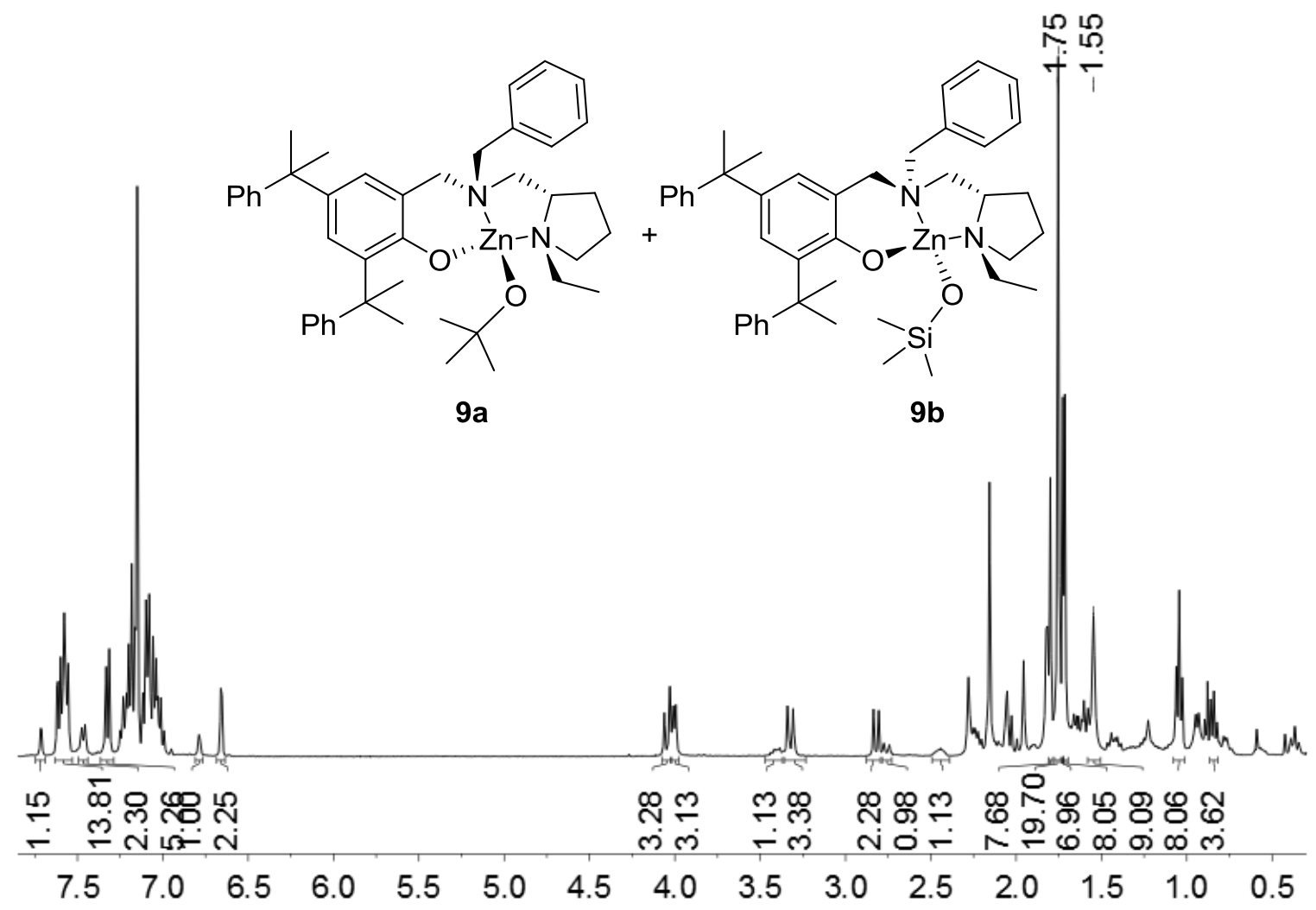

Figure S17. ${ }^{1} \mathrm{H}$ NMR spectrum $\left(\mathrm{C}_{6} \mathrm{D}_{6}, 400 \mathrm{MHz}, 20^{\circ} \mathrm{C}\right)$ of $\left[(S)-\mathrm{L}^{8}\right] \mathrm{ZnN}\left(\mathrm{OCMe}_{3}\right)(9)$.

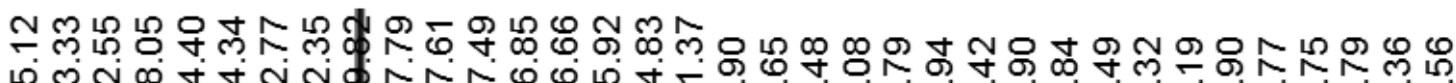
品

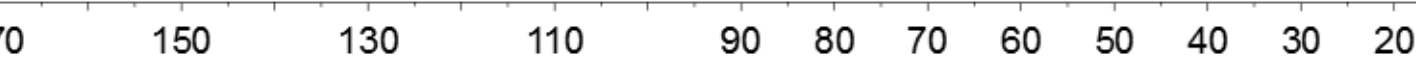

Figure S18. ${ }^{13} \mathrm{C}\left\{{ }^{1} \mathrm{H}\right\}$ NMR spectrum $\left(\mathrm{C}_{6} \mathrm{D}_{6}, 400 \mathrm{MHz}, 20{ }^{\circ} \mathrm{C}\right)$ of $\left[(S)-\mathrm{L}^{5}\right] \mathrm{ZnN}\left(\mathrm{OCMe}_{3}\right)(\mathbf{9})$. 


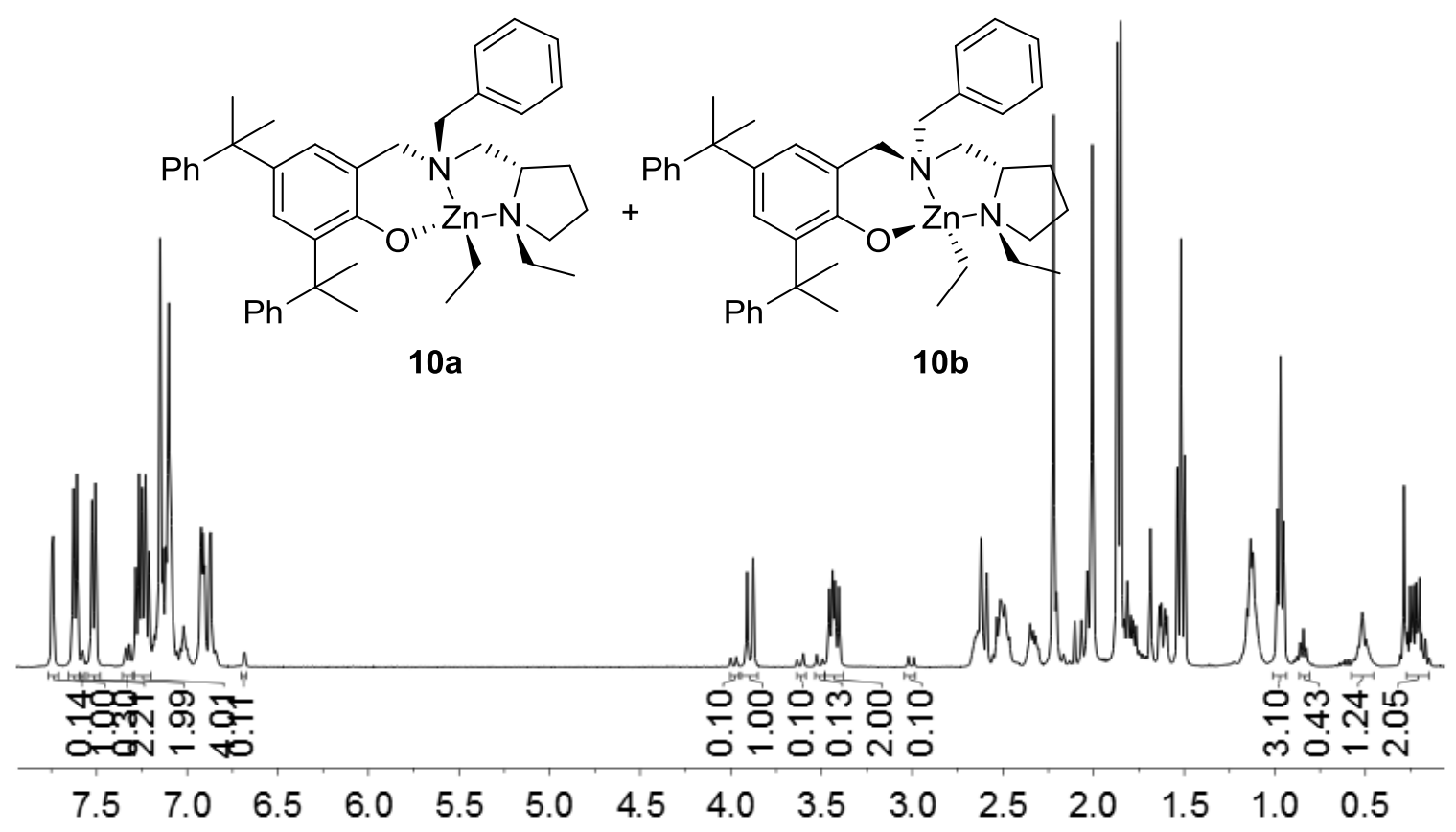

Figure S19. ${ }^{1} \mathrm{H}$ NMR spectrum $\left(\mathrm{C}_{6} \mathrm{D}_{6}, 400 \mathrm{MHz}, 20{ }^{\circ} \mathrm{C}\right)$ of $\left[(S)-\mathrm{L}^{8}\right] \mathrm{ZnEt}(\mathbf{1 0})$.

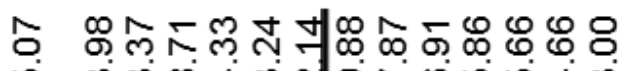

吕

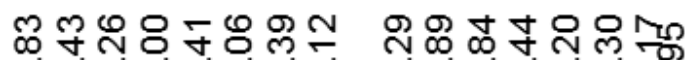

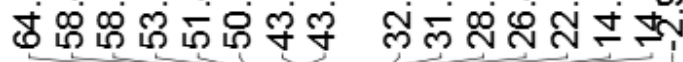

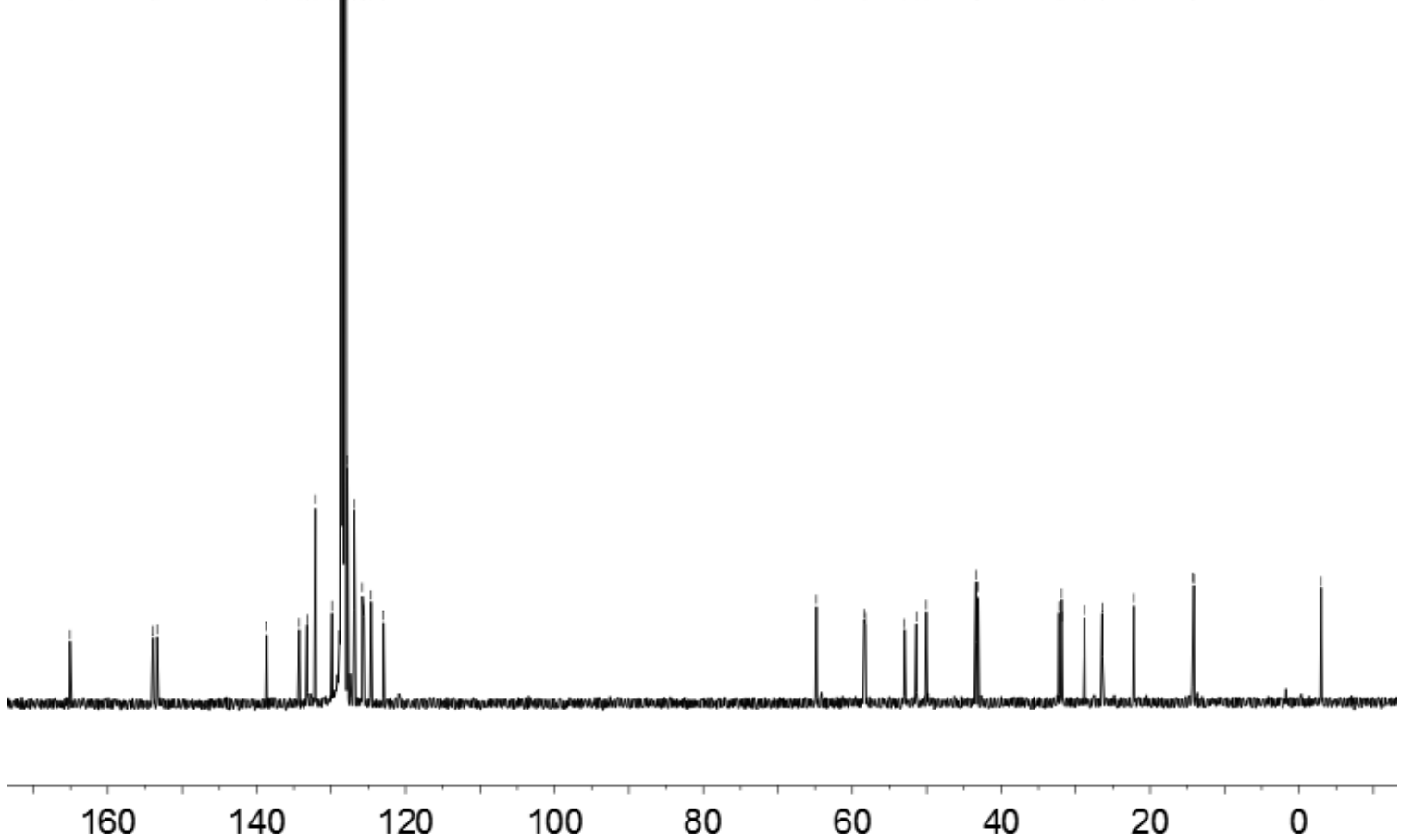

Figure S20. ${ }^{13} \mathrm{C}\left\{{ }^{1} \mathrm{H}\right\}$ NMR spectrum $\left(\mathrm{C}_{6} \mathrm{D}_{6}, 400 \mathrm{MHz}, 20{ }^{\circ} \mathrm{C}\right)$ of $\left[(S)-\mathrm{L}^{5}\right] \mathrm{ZnNEt}(\mathbf{1 0})$. 


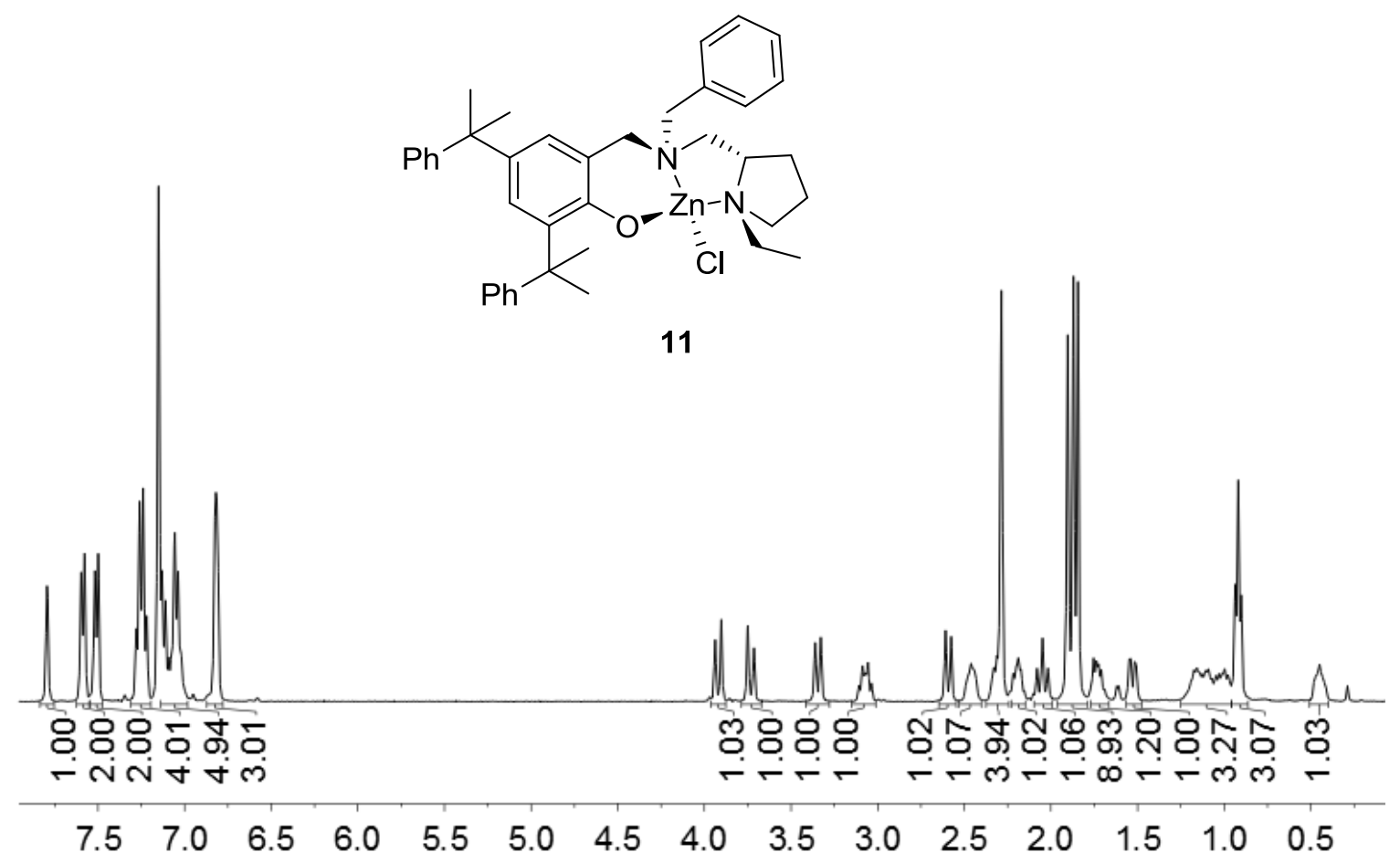

Figure S21. ${ }^{1} \mathrm{H} \mathrm{NMR}$ spectrum $\left(\mathrm{C}_{6} \mathrm{D}_{6}, 400 \mathrm{MHz}, 20{ }^{\circ} \mathrm{C}\right)$ of $\left[(S)-\mathrm{L}^{8}\right] \mathrm{ZnCl}(\mathbf{1 1})$.

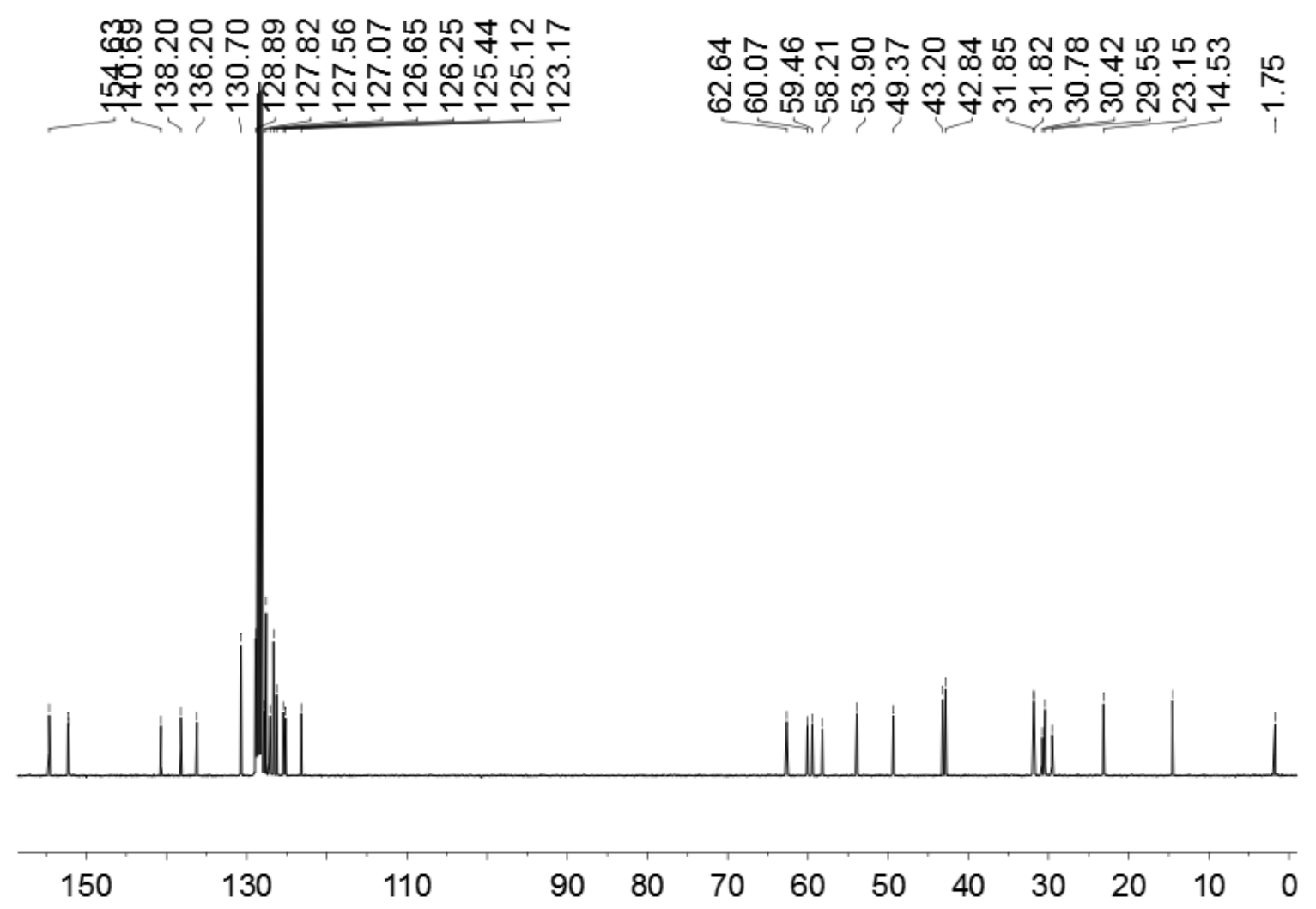

Figure S22. ${ }^{13} \mathrm{C}\left\{{ }^{1} \mathrm{H}\right\}$ NMR spectrum $\left(\mathrm{C}_{6} \mathrm{D}_{6}, 400 \mathrm{MHz}, 20{ }^{\circ} \mathrm{C}\right)$ of $\left[(S)-\mathrm{L}^{5}\right] \mathrm{ZnCl}(\mathbf{1 1})$. 


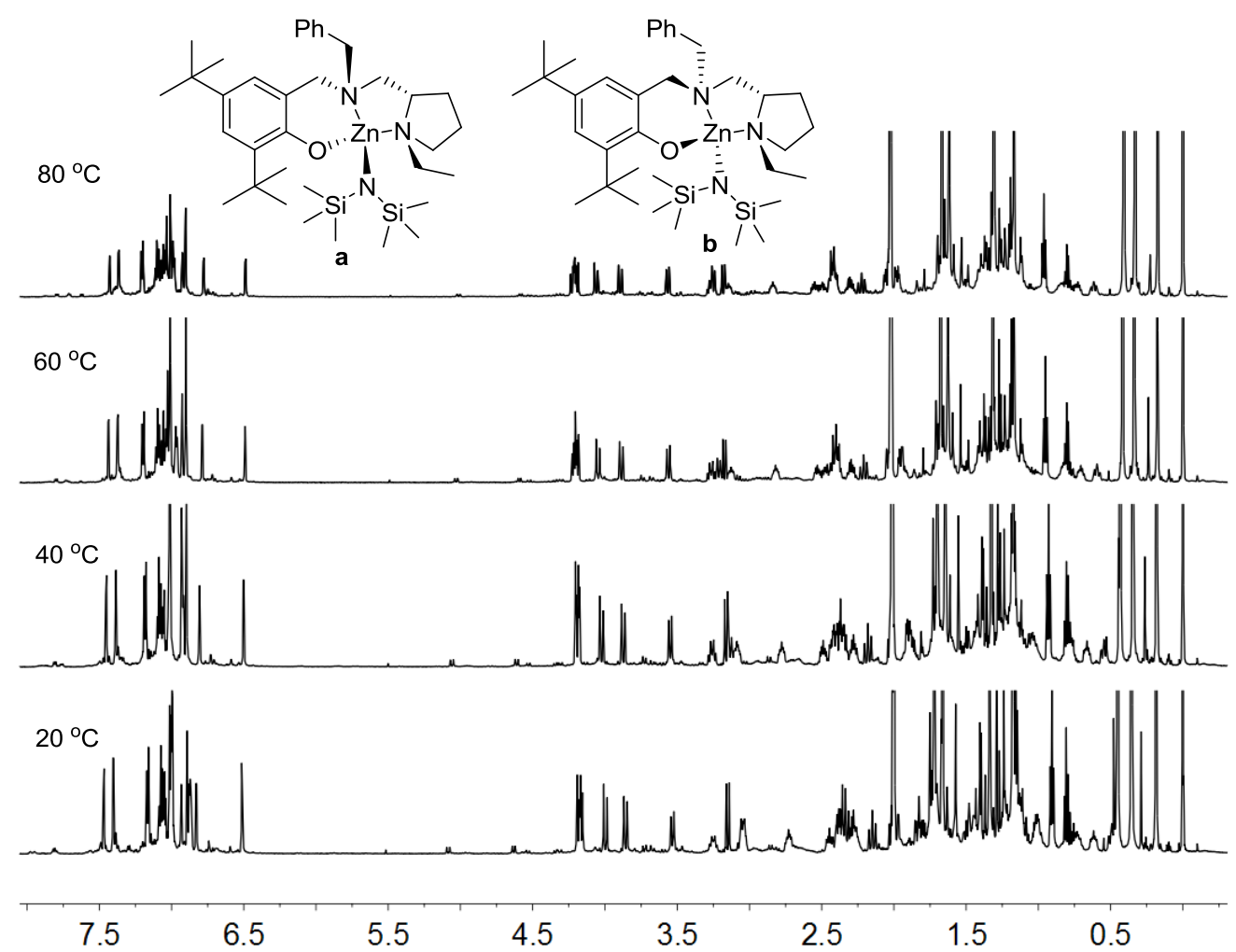

Figure S23 VT ${ }^{1} \mathrm{H}$ NMR spectra of zinc complex $3\left(\mathrm{C}_{7} \mathrm{D}_{8}, 500 \mathrm{MHz}\right)$

$80^{\circ} \mathrm{C}$

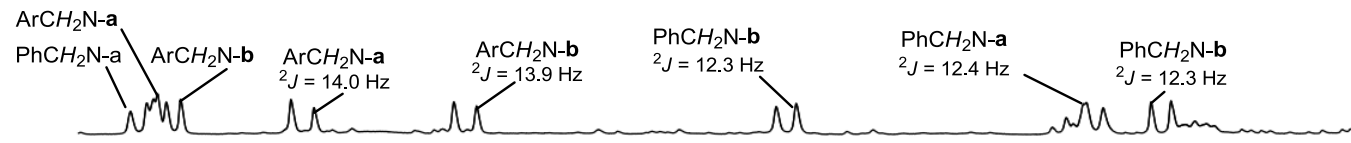

$60^{\circ} \mathrm{C}$

$\mathrm{ArCH}_{2} \mathrm{~N}-\mathrm{a}$

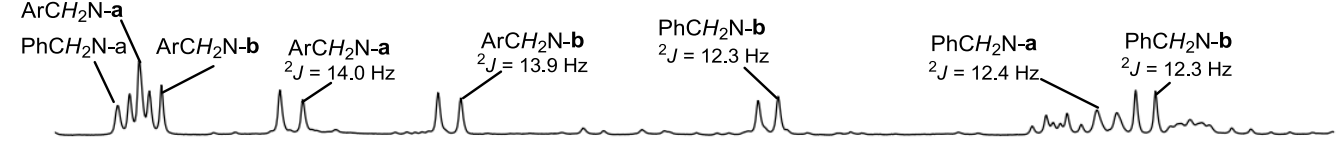

$40^{\circ} \mathrm{C}$

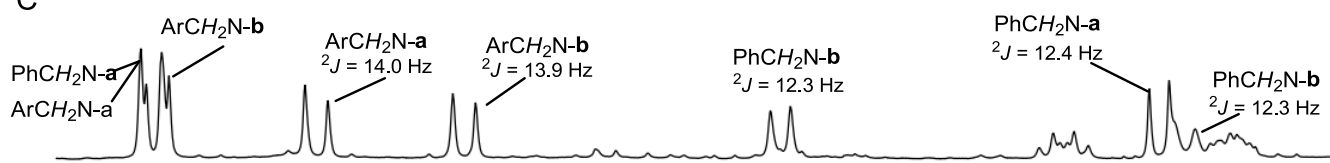

$20^{\circ} \mathrm{C}$

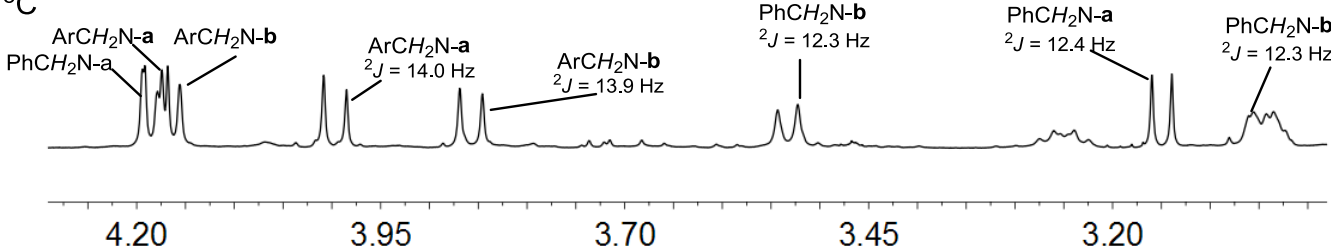

Figure S24 Selected VT ${ }^{1}$ H NMR spectra showing the benzyl proton region of zinc complex $\mathbf{3}$ $\left(\mathrm{C}_{7} \mathrm{D}_{8}, 500 \mathrm{MHz}\right)$ 


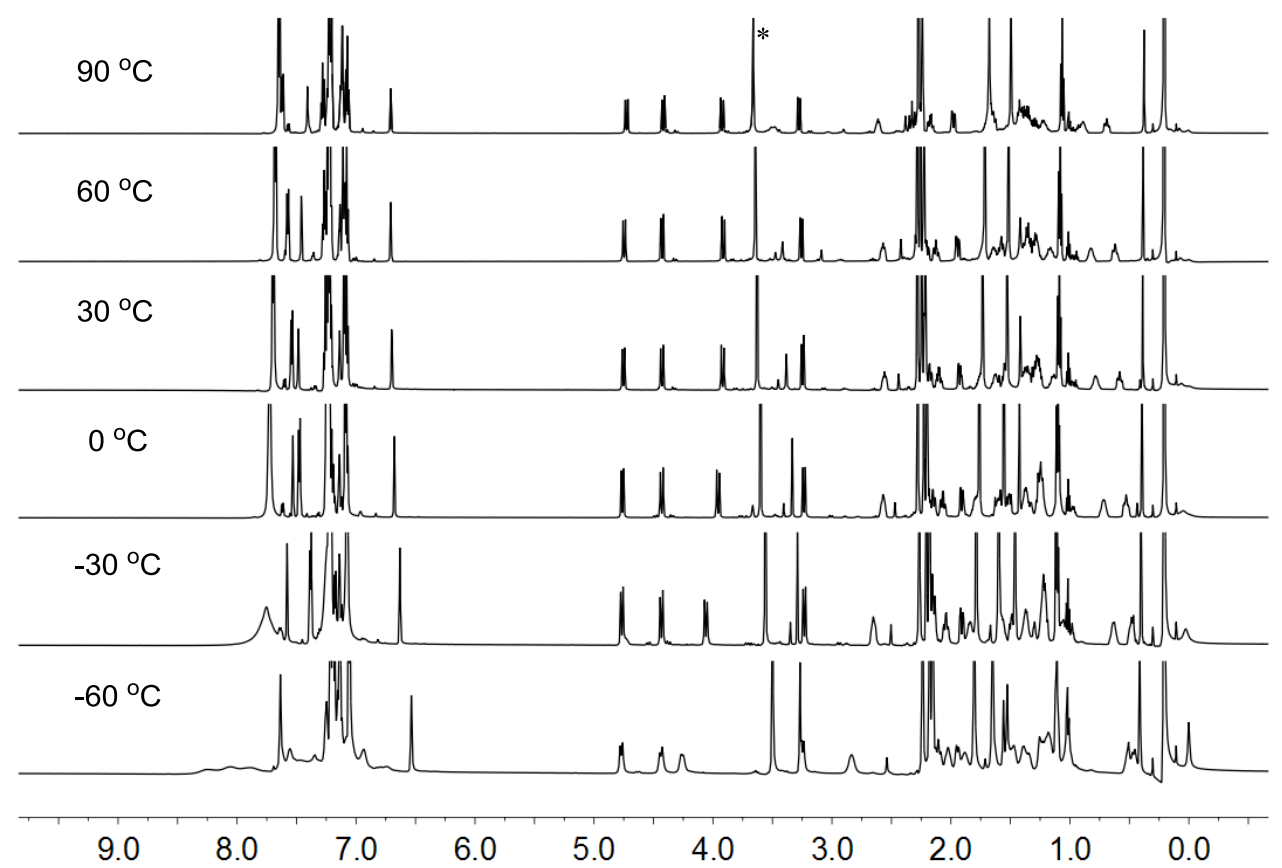

Figure S25 VT ${ }^{1} \mathrm{H}$ NMR spectra of zinc complex $\mathbf{1 3}\left(\mathrm{C}_{7} \mathrm{D}_{8}, 500 \mathrm{MHz}\right)$

\section{MALDI-TOF spectra of typical polymer samples}

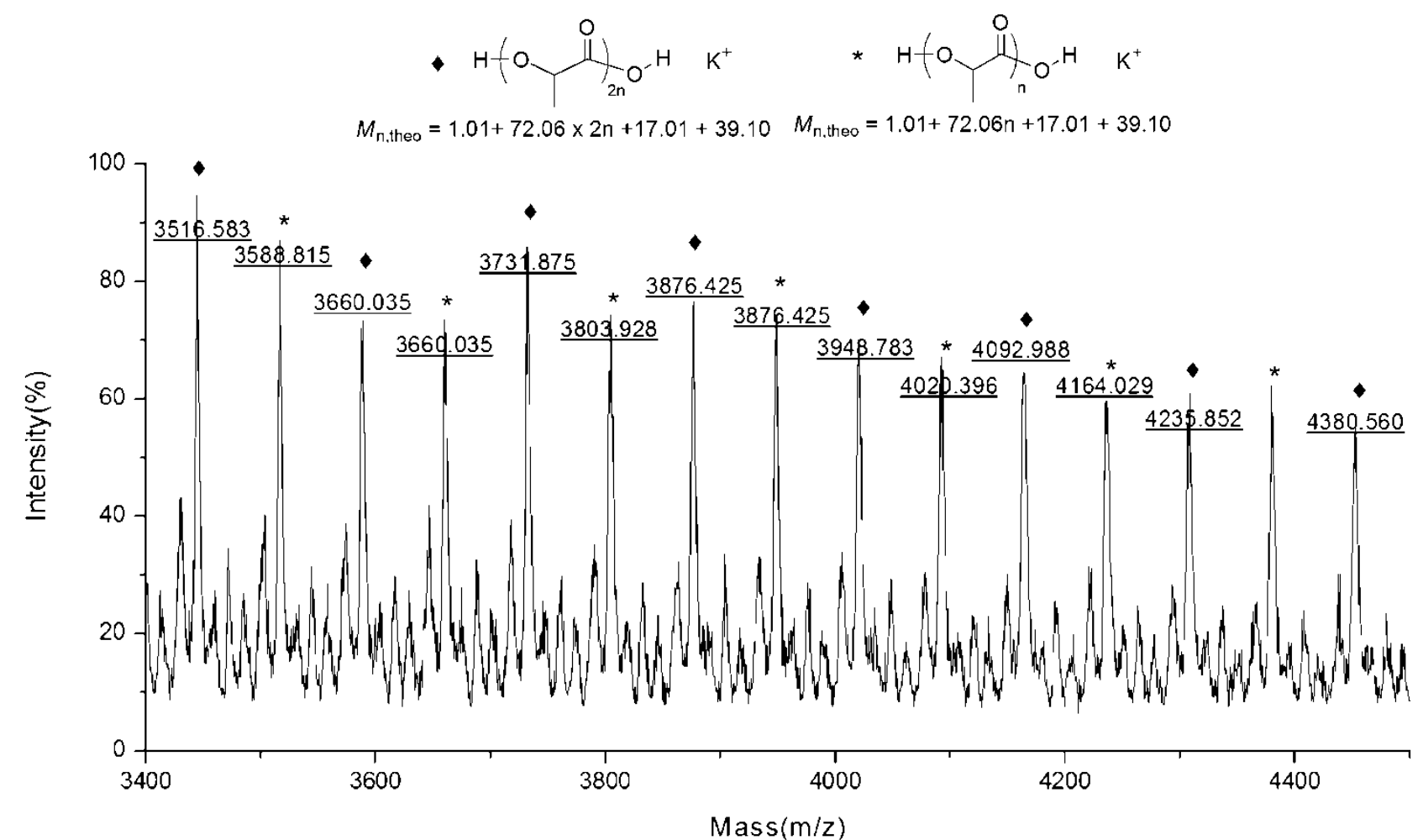

Figure S26. MALDI-TOF mass spectrum of PLA sample obtained with $[\mathrm{rac}-\mathrm{LA}]_{0} /[\mathbf{1 0}]_{0}=200,90 \%$ conversion. Theoretical molecular weights calculated according to the equation: $M_{\mathrm{n}}=\left(\mathrm{DP}_{\mathrm{n}} \times M_{\mathrm{w}} \mathrm{LA}\right)$ $+M_{\mathrm{w}} \mathrm{H}+M_{\mathrm{w}} \mathrm{OH}+M_{\mathrm{w}} \mathrm{K}$, where $\mathrm{DP}_{\mathrm{n}}$ is the degree of polymerization, $M_{\mathrm{w}} \mathrm{H}=1.01 \mathrm{~g} \cdot \mathrm{mol}^{-1}, M_{\mathrm{w}} \mathrm{LA}=$ $144.13 \mathrm{~g} \cdot \mathrm{mol}^{-1}, M_{\mathrm{w}} \mathrm{OH}=17.01 \mathrm{~g} \cdot \mathrm{mol}^{-1}$, and $M_{\mathrm{w}} \mathrm{K}=39.10 \mathrm{~g} \cdot \mathrm{mol}^{-1}$. 


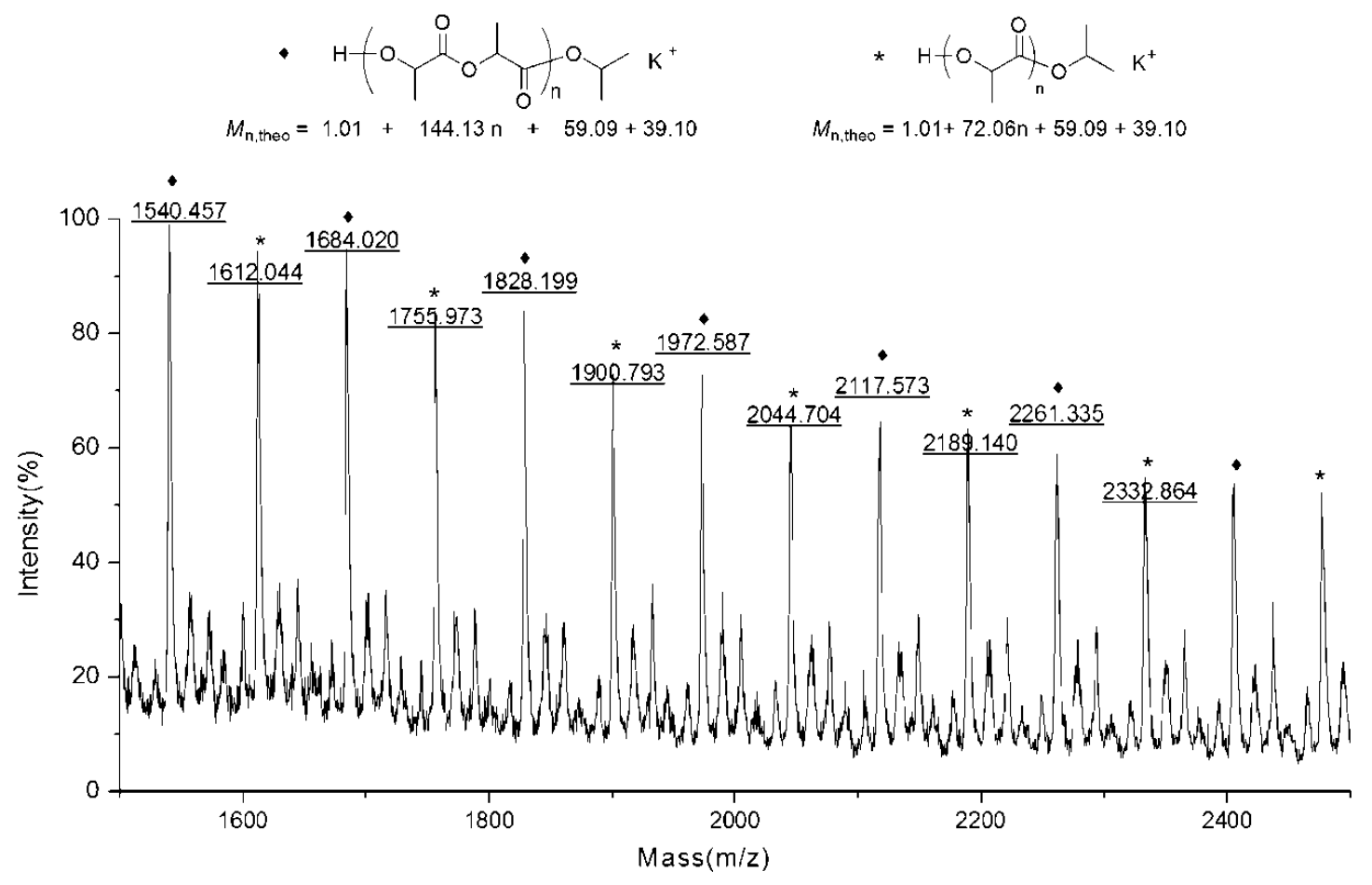

Figure S27. MALDI-TOF mass spectrum of PLA sample obtained with $[\mathrm{rac}-\mathrm{LA}]_{0} /\left[{ }^{i} \mathrm{PrOH}\right]_{0}[\mathbf{1 0}]_{0}=$ 200/1/1, 95\% conversion. Theoretical molecular weights calculated according to the equation: $M_{\mathrm{n}}=$ $\left(\mathrm{DP}_{\mathrm{n}} \times M_{\mathrm{w}} \mathrm{LA}\right)+M_{\mathrm{w}} \mathrm{H}+M_{\mathrm{w}} \mathrm{O} P r+M_{\mathrm{w}} \mathrm{K}$, where $\mathrm{DP}_{\mathrm{n}}$ is the degree of polymerization, $M_{\mathrm{w}} \mathrm{H}=1.01$ $\mathrm{g} \cdot \mathrm{mol}^{-1}, M_{\mathrm{w}} \mathrm{LA}=144.13 \mathrm{~g} \cdot \mathrm{mol}^{-1}, M_{\mathrm{w}} \mathrm{O}^{i} \mathrm{Pr}=59.09 \mathrm{~g} \cdot \mathrm{mol}^{-1}$, and $M_{\mathrm{w}} \mathrm{K}=39.10 \mathrm{~g} \cdot \mathrm{mol}^{-1}$.

\section{NMR studies of the reaction of zinc complexes and alcohols}
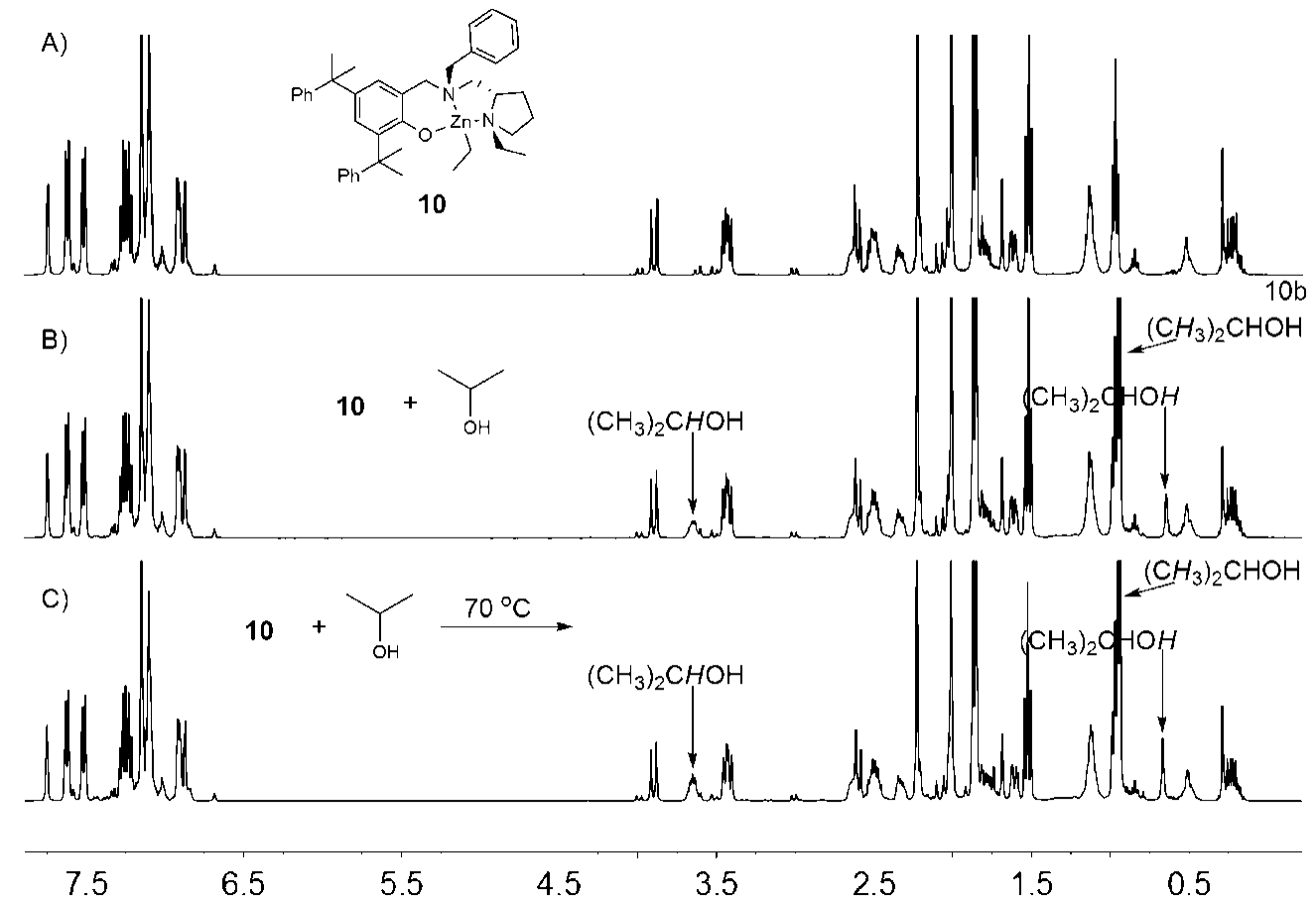

Figure S28. ${ }^{1} \mathrm{H}$ NMR spectra $\left(\mathrm{C}_{6} \mathrm{D}_{6}, 400 \mathrm{MHz}, 20{ }^{\circ} \mathrm{C}\right)$ of $\left.\mathrm{A}\right)\left[(S)-\mathrm{L}^{8}\right] \mathrm{ZnEt}(\mathbf{1 0})$; B) the NMR tube reaction of $\mathbf{1 0}$ and ${ }^{i} \mathrm{PrOH}$; C) the NMR tube reaction of $\mathbf{1 0}$ and ${ }^{i} \mathrm{PrOH}$ at $70{ }^{\circ} \mathrm{C}$ after $12 \mathrm{~h}$. 
A)

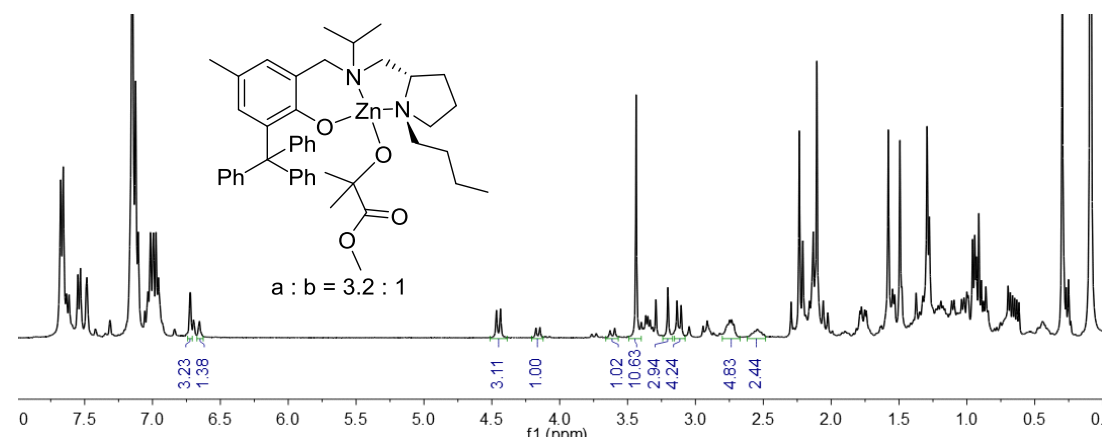

B)

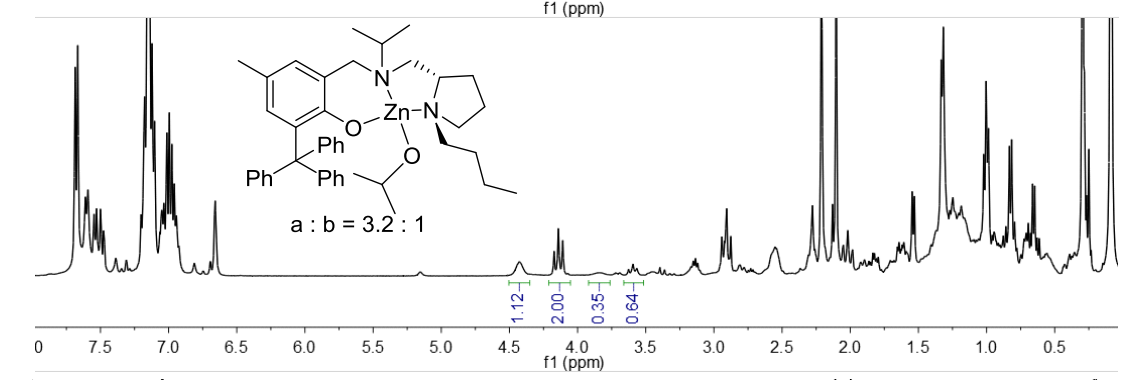

C)

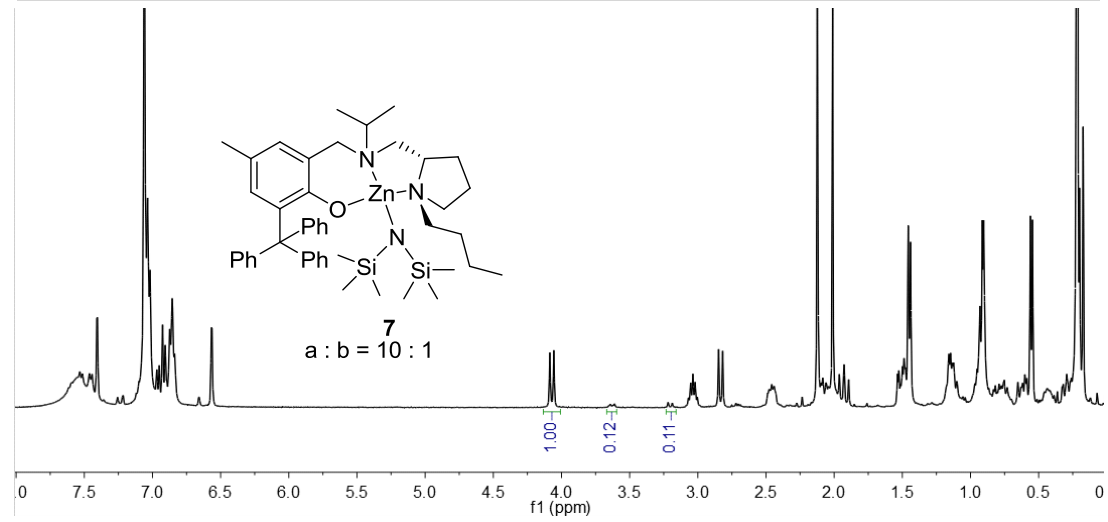

Figure S29. ${ }^{1} \mathrm{H}$ NMR spectra $\left(\mathrm{C}_{6} \mathrm{D}_{6}, 400 \mathrm{MHz}, 20{ }^{\circ} \mathrm{C}\right)$ of $\left.\mathrm{A}\right)$ the reaction mixture of $[(S)$ $\left.\mathrm{L}^{7}\right] \mathrm{ZnN}\left(\mathrm{SiMe}_{3}\right)_{2}(7)$ and $\left.\operatorname{HOCMe}{ }_{2} \mathrm{COOMe}(1: 1) ; \mathrm{B}\right)$ the reaction mixture of $\left[(S)-\mathrm{L}^{7}\right] \mathrm{ZnN}\left(\mathrm{SiMe}_{3}\right)_{2}(7)$ and ${ }^{i} \mathrm{PrOH}(1: 1)$; C) $\left[(S)-\mathrm{L}^{7}\right] \mathrm{ZnN}\left(\mathrm{SiMe}_{3}\right)_{2}(7)$.
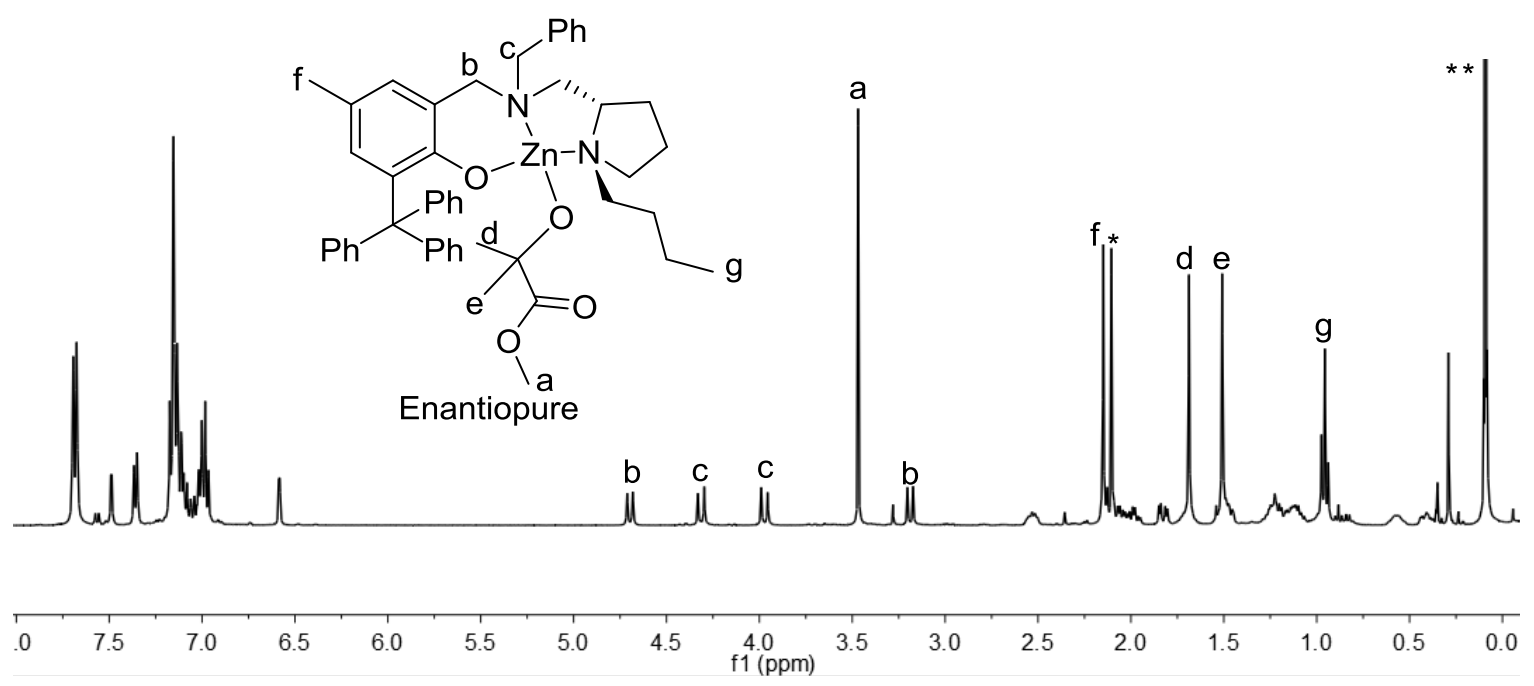

Figure S30. ${ }^{1} \mathrm{H}$ NMR spectra $\left(\mathrm{C}_{6} \mathrm{D}_{6}, 400 \mathrm{MHz}, 20{ }^{\circ} \mathrm{C}\right)$ reaction of $\left[(S)-\mathrm{L}^{13}\right] \mathrm{ZnN}\left(\mathrm{SiMe}_{3}\right)_{2}(\mathbf{1 3})+$ $\mathrm{HOCMe}_{2} \mathrm{COOMe}, *$ methyl of toluene; ** free $\mathrm{HN}\left(\mathrm{SiMe}_{3}\right)_{2}$ 
5. Microstructure analysis of poly(rac-lactide)s

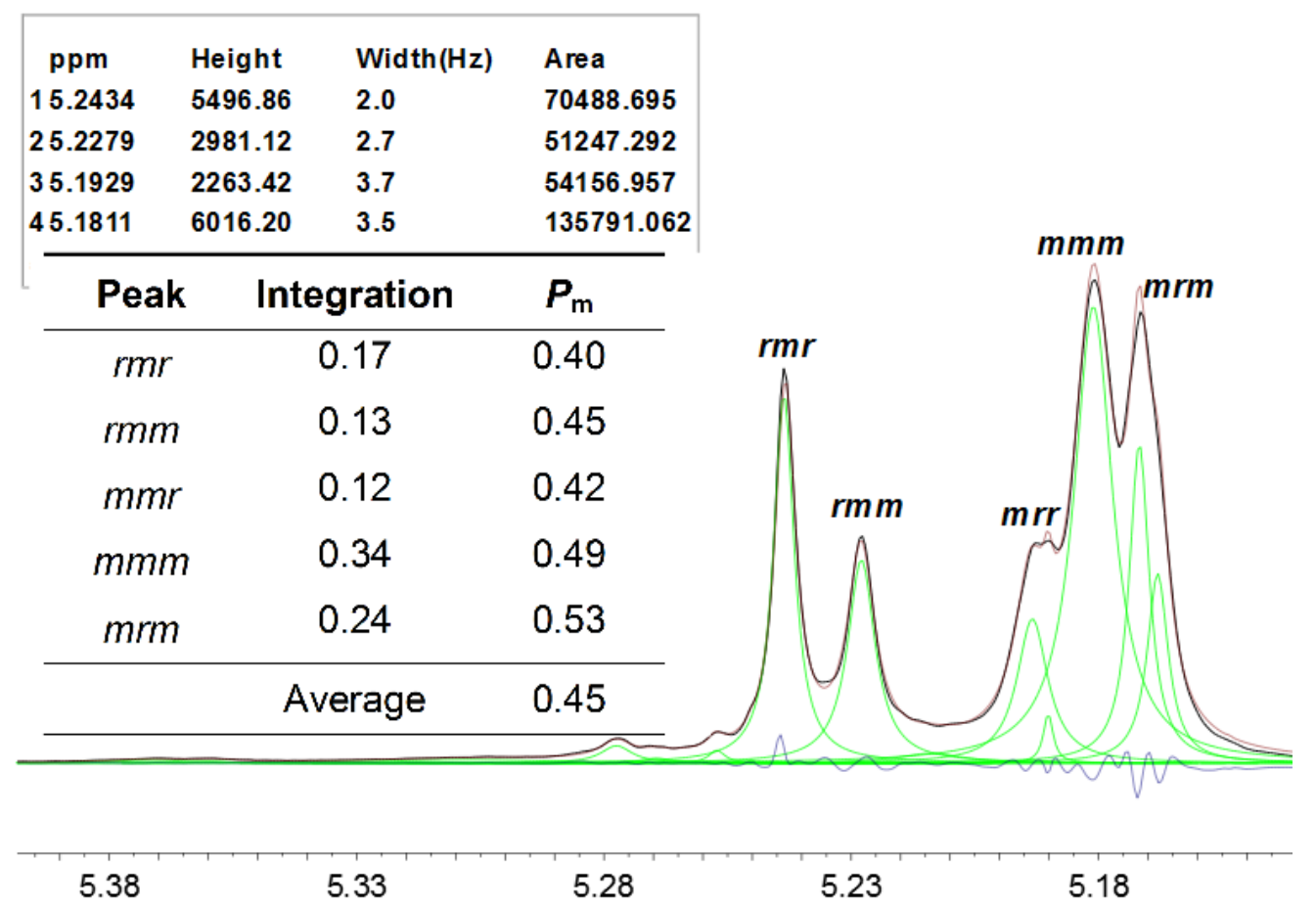

Figure S31. De-convoluted homonuclear decoupled ${ }^{1} \mathrm{H}$ NMR spectra of PLAs obtained from raclactide by using 1 as initiator in toluene at $25^{\circ} \mathrm{C}\left(\mathrm{CDCl}_{3}, 400 \mathrm{MHz}, P_{\mathrm{m}}=0.45\right)$.

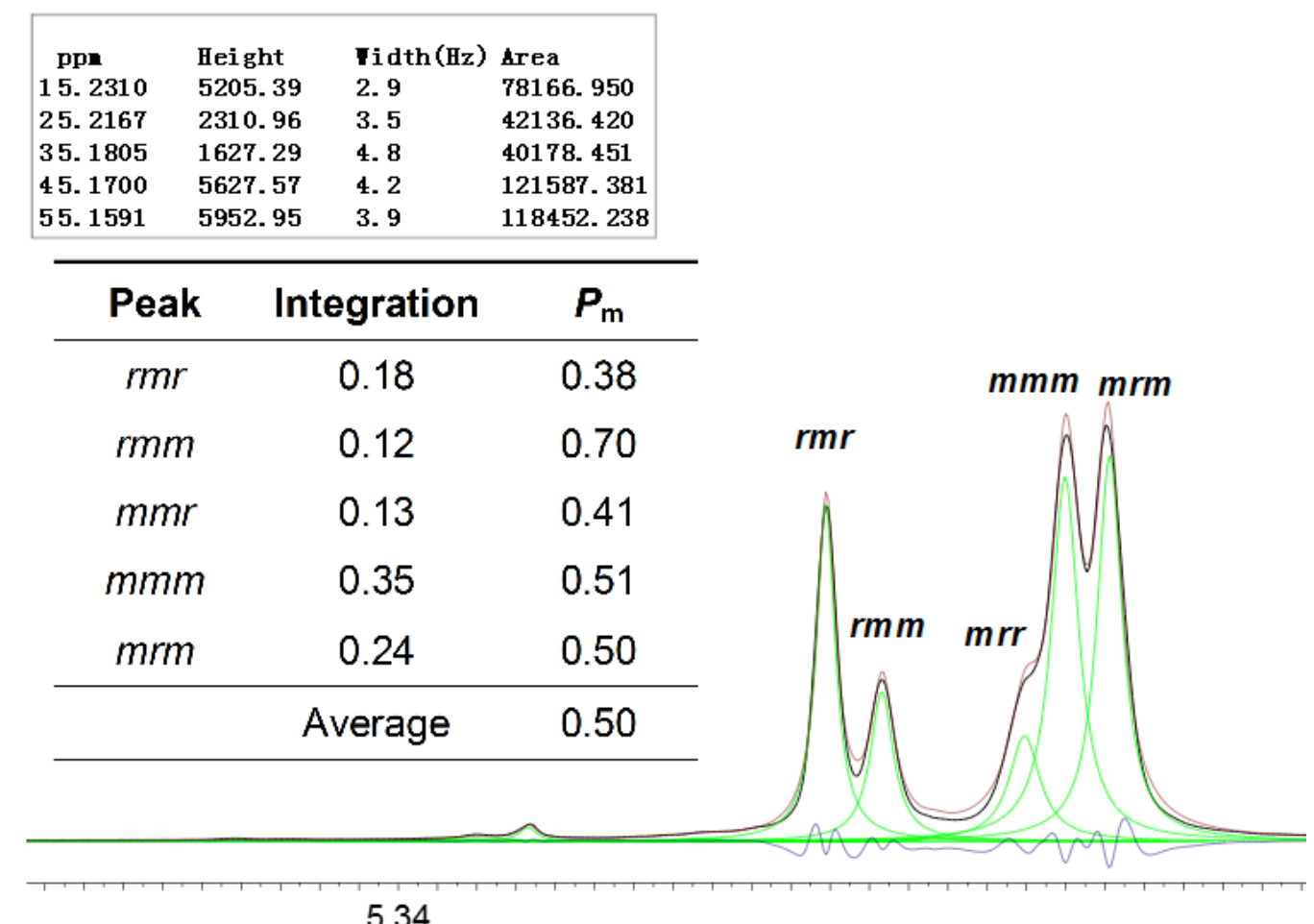

Figure S32. De-convoluted homonuclear decoupled ${ }^{1} \mathrm{H}$ NMR spectra of PLAs obtained from raclactide by using 2 as initiator in toluene at $25^{\circ} \mathrm{C}\left(\mathrm{CDCl}_{3}, 400 \mathrm{MHz}, P_{\mathrm{m}}=0.50\right)$. 


\begin{tabular}{|c|c|c|c|}
\hline ppm & \multirow{2}{*}{$\begin{array}{l}\text { Height } \\
301.34\end{array}$} & \multicolumn{2}{|c|}{ Width $(\mathrm{Hz})$ A rea } \\
\hline 15.1627 & & 1.9 & 3694.199 \\
\hline 25.1485 & 483.33 & 2.2 & 6834.771 \\
\hline 35.1136 & 302.10 & 3.0 & 5863.354 \\
\hline 45.1020 & 2181.70 & 3.2 & 44275.192 \\
\hline 55.0924 & 471.20 & 2.2 & 6600.804 \\
\hline \multicolumn{2}{|c|}{ Peak } & Integration & $P_{\mathrm{m}}$ \\
\hline \multicolumn{2}{|c|}{$r m r$} & 0.04 & 0.71 \\
\hline \multicolumn{2}{|c|}{$\mathrm{rmm}$} & 0.10 & 0.72 \\
\hline \multicolumn{2}{|c|}{$m m r$} & 0.08 & 0.77 \\
\hline \multicolumn{2}{|c|}{$\mathrm{mmm}$} & 0.63 & 0.74 \\
\hline \multicolumn{2}{|c|}{$\mathrm{mrm}$} & 0.16 & 0.70 \\
\hline \multicolumn{3}{|r|}{ Average } & 0.73 \\
\hline
\end{tabular}

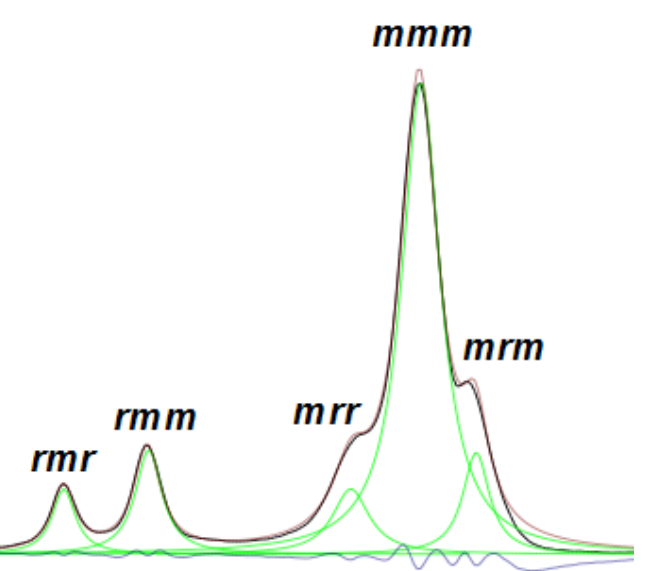

$\begin{array}{llllll}5.29 & 5.25 & 5.21 & 5.17 & 5.13 & 5.09\end{array}$

Figure S33. De-convoluted homonuclear decoupled ${ }^{1} \mathrm{H}$ NMR spectra of PLAs obtained from raclactide by using 3 as initiator in toluene at $25^{\circ} \mathrm{C}\left(\mathrm{CDCl}_{3}, 400 \mathrm{MHz}, P_{\mathrm{m}}=0.73\right)$.

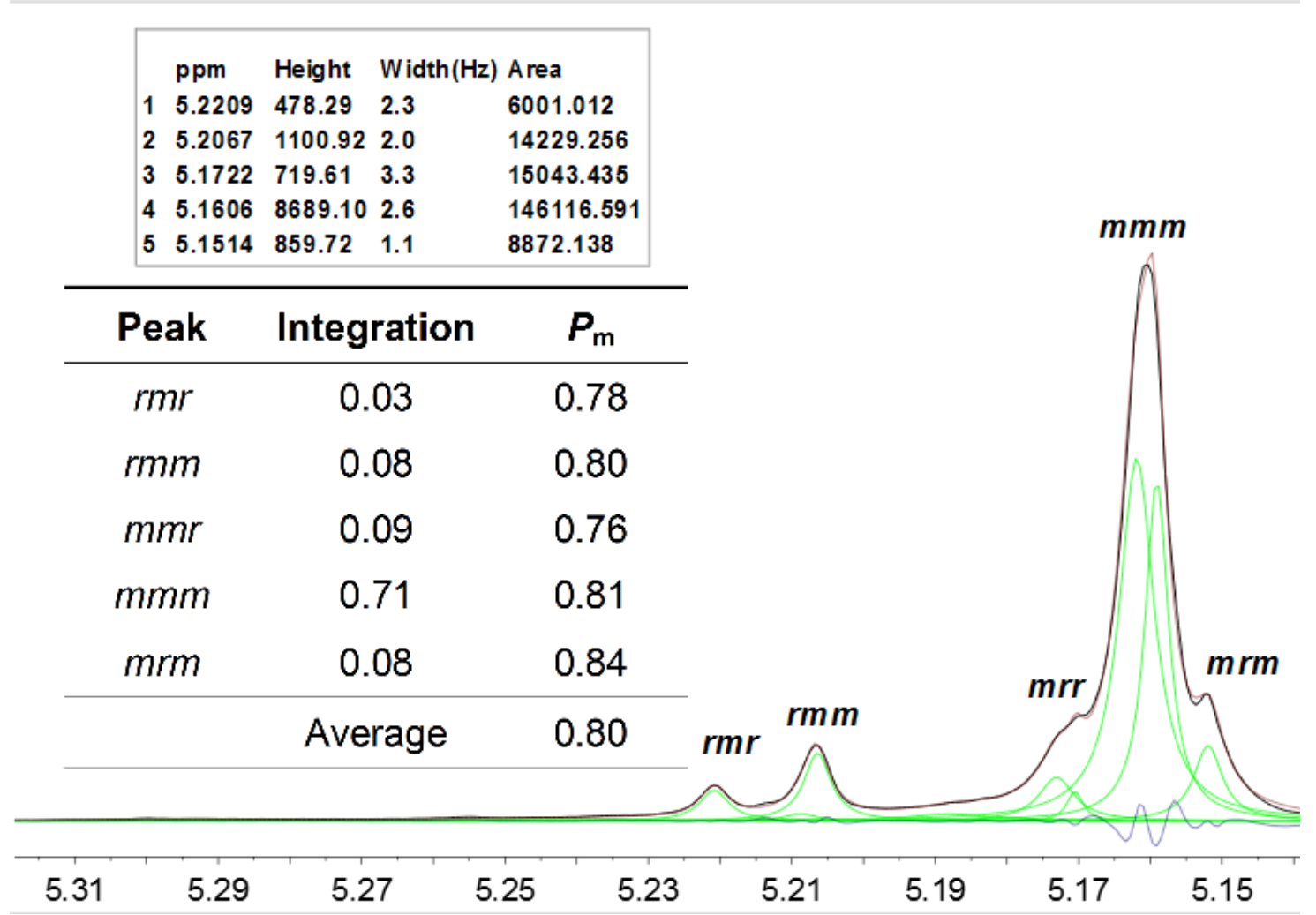

Figure S34. De-convoluted homonuclear decoupled ${ }^{1} \mathrm{H}$ NMR spectra of PLAs obtained from raclactide by using 4 as initiator in toluene at $25^{\circ} \mathrm{C}\left(\mathrm{CDCl}_{3}, 400 \mathrm{MHz}, P_{\mathrm{m}}=0.80\right)$. 


\begin{tabular}{|c|c|c|c|}
\hline ppm & Height & Width $(\mathrm{Hz})$ & Area \\
\hline 15.2322 & 94.08 & 2.2 & 1345.513 \\
\hline 25.2178 & 123.97 & 2.2 & 1718.081 \\
\hline 35.1816 & 116.03 & 5.3 & 3918.502 \\
\hline 45.1713 & 585.00 & 2.9 & 10857.477 \\
\hline 55.1619 & 132.83 & 2.3 & 1934.048 \\
\hline \multicolumn{2}{|c|}{ Peak } & ntegration & $\boldsymbol{P}_{\mathrm{m}}$ \\
\hline \multicolumn{2}{|c|}{$r m r$} & 0.06 & 0.67 \\
\hline \multicolumn{2}{|c|}{$\mathrm{rmm}$} & 0.10 & 0.72 \\
\hline \multicolumn{2}{|c|}{$m m r$} & 0.08 & 0.73 \\
\hline \multicolumn{2}{|c|}{$\mathrm{mmm}$} & 0.60 & 0.70 \\
\hline \multicolumn{2}{|c|}{$\mathrm{mrm}$} & 0.16 & 0.68 \\
\hline \multicolumn{3}{|r|}{ Average } & 0.70 \\
\hline
\end{tabular}

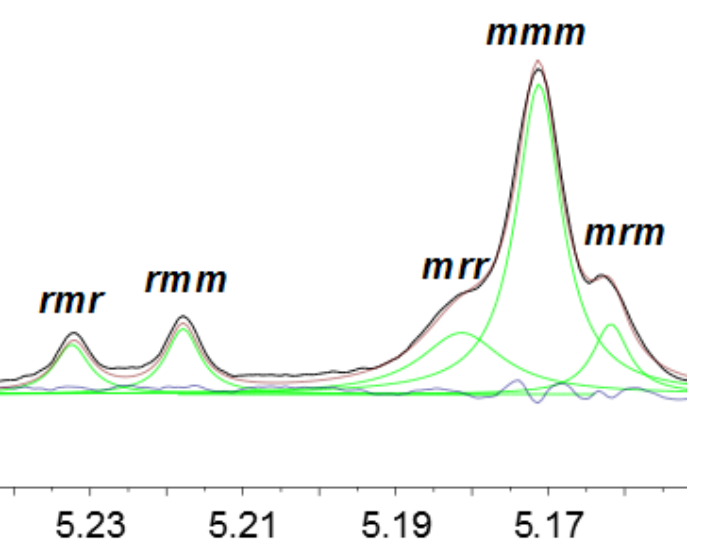

Figure S35. De-convoluted homonuclear decoupled ${ }^{1} \mathrm{H}$ NMR spectra of PLAs obtained from raclactide by using 5 as initiator in toluene at $25^{\circ} \mathrm{C}\left(\mathrm{CDCl}_{3}, 400 \mathrm{MHz}, P_{\mathrm{m}}=0.70\right)$.

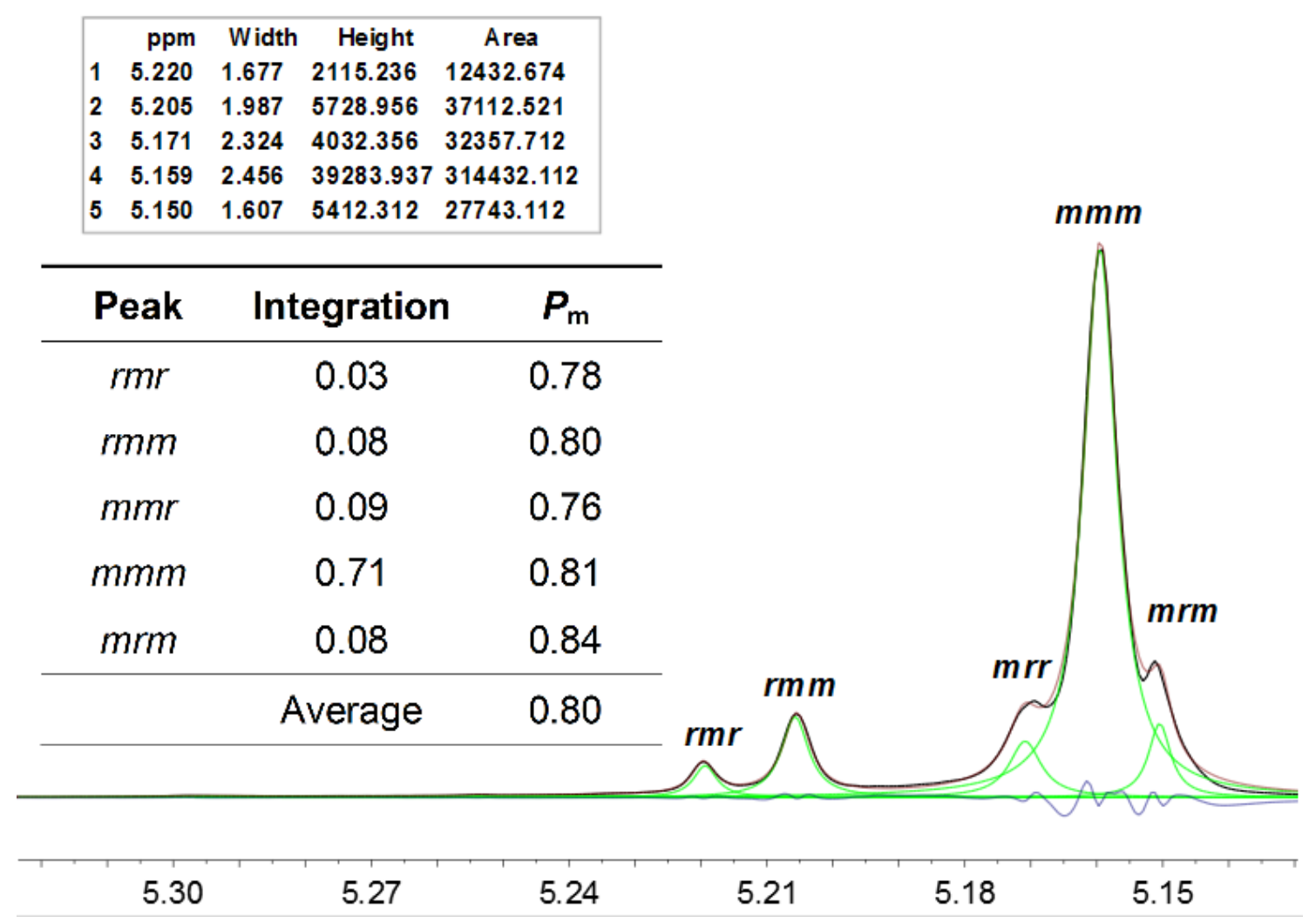

Figure S36. De-convoluted homonuclear decoupled ${ }^{1} \mathrm{H}$ NMR spectra of PLAs obtained from raclactide by using 6 as initiator in toluene at $25^{\circ} \mathrm{C}\left(\mathrm{CDCl}_{3}, 400 \mathrm{MHz}, P_{\mathrm{m}}=0.80\right)$. 


\begin{tabular}{|c|c|c|c|}
\hline ppm & Height & Width $(\mathrm{Hz})$ & Area \\
\hline 15.2324 & 2717.76 & 2.5 & 44354.216 \\
\hline 25.2180 & 2459.16 & 3.1 & 49361.376 \\
\hline 35.1833 & 2408.77 & 4.0 & 61525.243 \\
\hline 45.1714 & 6088.29 & 3.5 & 138185.556 \\
\hline 55.1620 & 4448.79 & 2.9 & 83857.808 \\
\hline \multicolumn{2}{|c|}{ Peak } & Integration & $P_{\mathrm{m}}$ \\
\hline \multicolumn{2}{|c|}{$\mathrm{rmr}$} & 0.12 & 0.50 \\
\hline \multicolumn{2}{|c|}{$\mathrm{rmm}$} & 0.13 & 0.65 \\
\hline \multicolumn{2}{|c|}{$m m r$} & 0.13 & 0.46 \\
\hline \multicolumn{2}{|c|}{$\mathrm{mmm}$} & 0.41 & 0.55 \\
\hline \multicolumn{2}{|c|}{$\mathrm{mrm}$} & 0.21 & 0.58 \\
\hline \multicolumn{3}{|r|}{ Average } & 0.55 \\
\hline
\end{tabular}

$m m m$

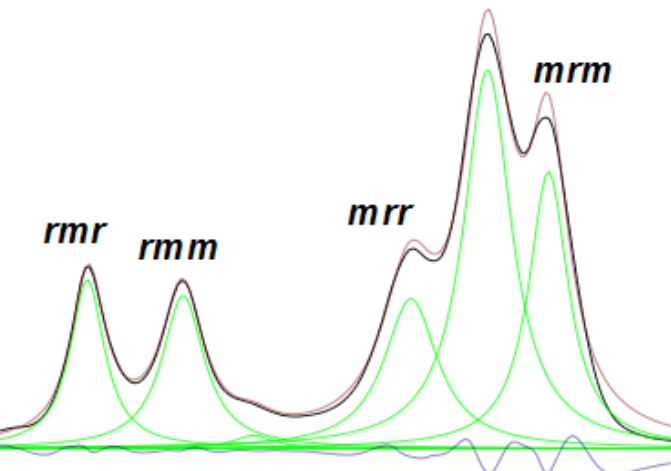

$5.23 \quad 5.20$

5.17

Figure S37. De-convoluted homonuclear decoupled ${ }^{1} \mathrm{H}$ NMR spectra of PLAs obtained from raclactide by using 7 as initiator in toluene at $25^{\circ} \mathrm{C}\left(\mathrm{CDCl}_{3}, 400 \mathrm{MHz}, P_{\mathrm{m}}=0.55\right)$.

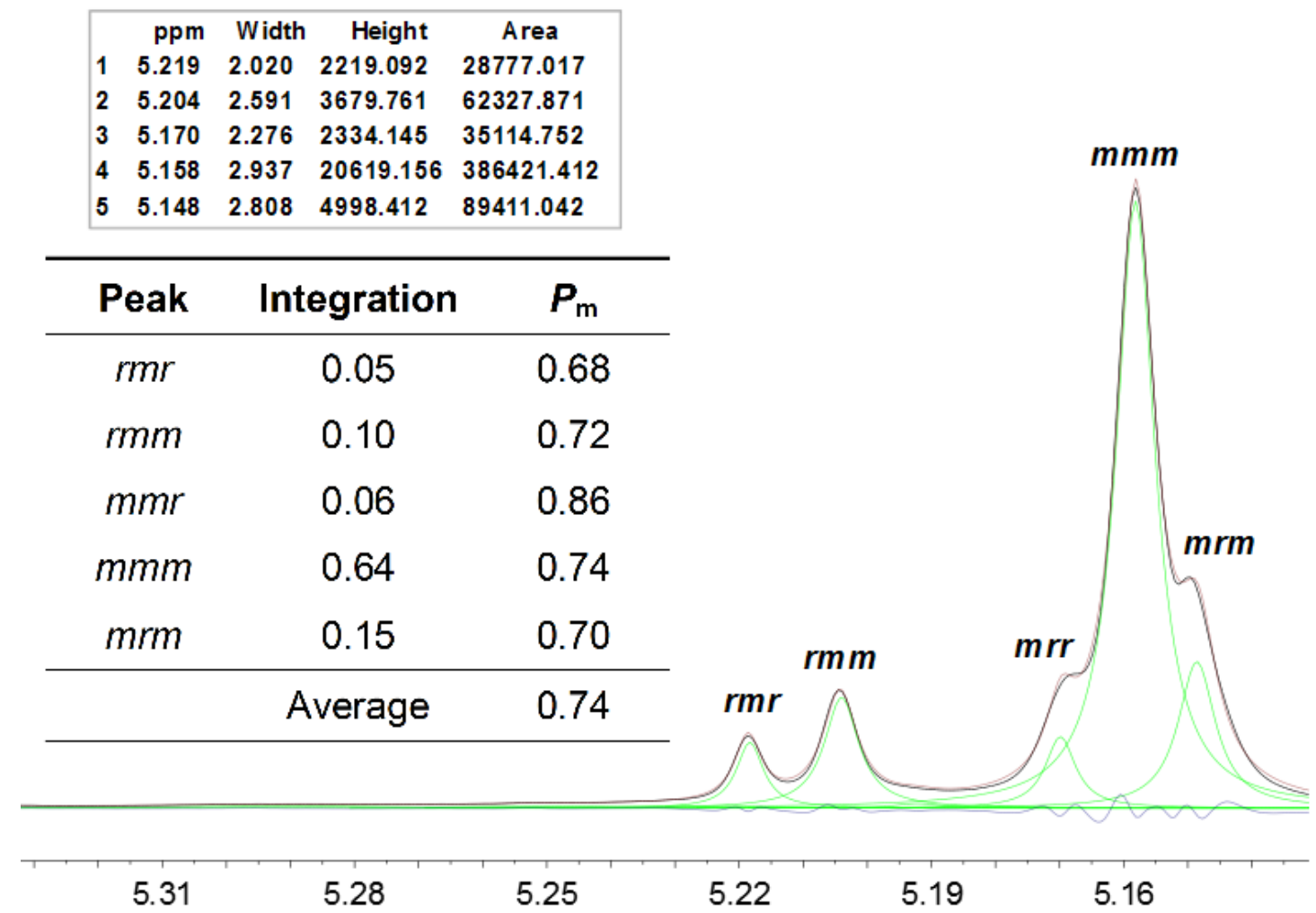

Figure S38. De-convoluted homonuclear decoupled ${ }^{1} \mathrm{H}$ NMR spectra of PLAs obtained from raclactide by using 8,9 and 10 as initiator in toluene at $25^{\circ} \mathrm{C}\left(\mathrm{CDCl}_{3}, 400 \mathrm{MHz}, P_{\mathrm{m}}=0.74\right)$. 


\section{References}

[S1] a) G. S. Lannoye, S. M. Moerlein, D.Parkinson, M. J. Welch, J. Med. Chem. 1990, 33, 2430.

b) M. B. Sassaman, J. Giovanelli, V. K. Soodc, W. C. Eckelman, Bioorg. Med. Chem. 1998, $6,1759$. 